\title{
Lattice Boltzmann Simulations of 2D Laminar Flows past Two Tandem Cylinders
}

\author{
Alberto Mussa ${ }^{a}$, Pietro Asinari ${ }^{\mathrm{a}, *}$, and Li-Shi Luo ${ }^{\mathrm{b}}$ \\ ${ }^{a}$ Department of Energetics, Politecnico di Torino, Torino 10129, Italy \\ Tel.: +39 (011) 090-4520, Fax: +39 (011) 090-4499 \\ ${ }^{\mathrm{b}}$ Department of Mathematics $\&$ Statistics and Center for Computational Sciences \\ Old Dominion University, Norfolk, VA 23529, USA
}

\begin{abstract}
We apply the lattice Boltzmann equation (LBE) with multiple-relaxation-time (MRT) collision model to simulate laminar flows in two-dimensions (2D). In order to simulate flows in an unbounded domain with the LBE method, we need to address two issues: stretched non-uniform mesh and in-flow and out-flow boundary conditions. We use the interpolated grid stretching method to address the need of non-uniform mesh. We demonstrate that various in-flow and out-flow boundary conditions can be easily and consistently realized with the MRT-LBE. The MRT-LBE with nonuniform stretched grids is first validated with a number of test cases: the Poiseuille flow, the flow past a cylinder asymmetrically placed in a channel, and the flow past a cylinder in an unbounded domain. We use the LBE method to simulate the flow past two tandem cylinders in a unbounded domain with $\mathrm{Re}=100$. Our results agree well with existing ones. Through this work we demonstrate the effectiveness of the MRT-LBE method with grid stretching.
\end{abstract}

Key words: lattice Boltzmann equation, boundary conditions, grid stretching, flow past tandem cylinders

\section{Introduction}

In recent years the lattice Boltzmann equation (LBE) has become a viable means for computational fluid dynamics (CFD) (cf. [1] and references therein).

* Corresponding author.

Email addresses: pietro.asinari@polito.it (Pietro Asinari), lluo@odu.edu

(Li-Shi Luo).

URL: http://www. lions.odu.edu/ lluo (Li-Shi Luo). 
As opposed to conventional CFD methods based on direct discretizations of the Navier-Stokes equations, the LBE method is derived from the Boltzmann equation and kinetic theory [2,3]. The kinetic origin of the LBE method differentiates it from conventional CFD methods in several ways. In the LBE method, one deals with the discretized particle velocity distribution functions $\left\{f_{i}\right\}$ instead of the hydrodynamic variables. Therefore, one must also deal with the boundary conditions for the distribution functions $\left\{f_{i}\right\}$ instead of that for the hydrodynamic variables. This kinetic nature of boundary conditions in the LBE method has some times caused confusions and the boundary conditions in the LBE is still a topic of active research.

The most popular LBE implementation of the Dirichlet boundary conditions on the flow velocity $\boldsymbol{u}$ or pressure $p$ is the bounce-back (BB) boundary condition $(\mathrm{BC})$. When the bounce-back boundary conditions applied with the simple lattice Bhatnagar-Gross-Krook (BGK) equation with one single relaxation parameter $\tau$, the exact location where the Dirichlet boundary conditions for $\boldsymbol{u}$ or $p$ are satisfied depends on the viscosity $\nu$ (or the relaxation parameter $\tau)$ [4-8]. This problem in the lattice BGK (LBGK) equation with bounce-back BCs has generate numerous papers (e.g., [9-15]). Unfortunately, none of these works provides a rigorous analysis of or a systematic remedy to the problem due to inaccurate BB-BCs with the LBGK model, in spite of the fact that this problem can be analyzed $[4-7]$ and removed $[16,7,8]$ by using the lattice Boltzmann equation with multiple-relaxation-time (MRT) models [17$20]$ with improved boundary conditions $[16,7,8]$. It should also be noted that asymptotic analysis $[21,22]$ has been used to address this problem in LBE [23].

In addition to flow-solid boundary conditions, LBE implementation of various inflow and outflow boundary conditions have been considered. Various approaches have been proposed previously in the context of the LBGK equation $[9,10,13,14]$. In flow simulations, boundary conditions only specify the values of hydrodynamic variables at boundaries. However, hydrodynamic boundary conditions are insufficient for the lattice Boltzmann equation, because the distribution functions $\left\{f_{i}\right\}$ have non-equilibrium moments which are not specified by the values of hydrodynamic variables. Similarly, hydrodynamic initial conditions are insufficient to completely specify the LBE initial conditions but can be solved by an iterative procedure [24]. In this work we will demonstrate the inflow and outflow boundary conditions of either Dirichlet or Neumann type can be easily and consistently realized with the MRT-LBE method.

The LBE method usually employs uniform Cartesian meshes in both two and three dimensions. To be computationally efficient, non-uniform and adaptive meshes must be used. To this end, two approaches have been used in the LBE: the grid refinement $[15,25,26]$ and the interpolated grid stretching $[27,2,28]$. In grid refinement, Cartesian meshes are used, and grid spacing is divided by an integer, which is usually a power of 2 , to the next refined grid level. 
Interpolations are used at the interface between two connected meshes of different grid spacings, and at solid boundaries [29,8]. With the interpolated grid stretching method, one can use body-fitted meshes [30,31]. Except at flowsolid boundaries, interpolations have to be used throughout the entire mesh where the discretized distribution functions $\left\{f_{i}\right\}$ cannot be propagated exactly from one grid to another in the advection process. The simple bounce-back boundary conditions can be used with body-fitted meshes. We will use the latter approach, i.e., the interpolated grid stretching, in the present work.

In this paper we intend to use the LBE method to simulate laminar flows past two tandem cylinders in a unbounded domain in two-dimensions (2D). We shall restrict ourselves to the athermal (or isothermal) LBE models, in which the internal energy is not a conserved quantity. The main objective of this work is to investigate the effectiveness of the MRT-LBE with nonuniformly stretched grids for flow simulations. Implementations of inflow and outflow boundary conditions with the MRT-LBE method will also be validated through a number of test cases.

The remainder of this paper is organized as follows. We discuss the LBE method in Section 2, including descriptions of the MRT-LBE method, the interpolated bounce-back boundary conditions for arbitrary curved boundaries, inflow and outflow boundary conditions, force evaluation at flow-solid boundaries, and non-uniform grid stretching method. To validate the grid stretching method and inflow and outflow boundary conditions, we provide a number of test cases in Section 3. These test cases include: the Poiseuille flow, the flow past a cylinder asymmetrically placed in a channel at the Reynolds number Re $=20$ and 100 [32], and the flow past a cylinder in a unbounded domain. In Section 4 we present the LBE results for the flow past two tandem cylinders in a unbounded domain with $\mathrm{Re}=100$ [33-40]. We will compare our results with existing ones obtained with conventional Navier-Stokes solvers $[33,37,38]$. Finally we conclude the paper with Section 5.

\section{Numerical method}

\subsection{Multiple-relaxation-time lattice Boltzmann method}

We will use the lattice Boltzmann equation with the multiple-relaxation-time (MRT) collision model [17,18,20],

$$
\mathbf{f}\left(\boldsymbol{x}_{j}+\boldsymbol{c} \delta t, t_{n}+\delta t\right)=\mathbf{f}\left(\boldsymbol{x}_{j}, t_{n}\right)-\mathbf{M}^{-1} \cdot \hat{\mathrm{S}} \cdot\left[\mathbf{m}-\mathbf{m}^{(\mathrm{eq})}(\rho, \boldsymbol{u})\right]\left(\boldsymbol{x}_{j}, t_{n}\right),
$$


where $\rho$ and $\boldsymbol{u}$ are the macroscopic density and velocity respectively, the boldface symbols such as $\mathbf{f}$ denote $Q$-tuple vectors, and $Q$ is the number of discrete velocities:

$$
\begin{aligned}
& \mathbf{f}:=\left(f_{0}, f_{1}, \ldots, f_{Q-1}\right)^{\top}, \\
& \mathbf{f}\left(\boldsymbol{x}_{j}+\boldsymbol{c} \delta_{t}\right):=\left(f_{0}\left(\boldsymbol{x}_{j}\right), f_{1}\left(\boldsymbol{x}_{j}+\boldsymbol{c}_{1} \delta_{t}\right), \ldots, f_{Q-1}\left(\boldsymbol{x}_{j}+\boldsymbol{c}_{Q-1} \delta_{t}\right)\right)^{\top}, \\
& \mathbf{m}:=\left(m_{0}, m_{1}, \ldots, m_{Q-1}\right)^{\top}, \\
& \mathbf{m}^{\text {(eq) }}:=\left(m_{0}^{\text {(eq) }}, m_{1}^{\text {(eq) }}, \ldots, m_{Q-1}^{(\text {eq })}\right)^{\top},
\end{aligned}
$$

where $\mathrm{T}$ denotes the transpose operator.

We will use the nine velocity model (D2Q9 model), of which the discrete velocities are: $\boldsymbol{c}_{0}=(0,0), \boldsymbol{c}_{i}=( \pm 1,0) c$ and $(0, \pm 1) c$, for $i=1-4$, and $\boldsymbol{c}_{i}=( \pm 1, \pm 1) c$, for $i=5-8$, where $c:=\delta_{x} / \delta_{t}$, and $\delta_{x}$ and $\delta_{t}$ are the lattice constant (or grid spacing) and time step size, respectively $[2,3,18]$.

With the following specific order of the moments [18]:

$$
\mathbf{m}:=\left(\rho, e, \epsilon, u_{x}, q_{x}, u_{y}, q_{y}, p_{x x}, p_{x y}\right)^{\top},
$$

where $\rho$ is the fluid density (zeroth-order velocity moment), $\boldsymbol{u}$ the velocity (first-order moments), $p_{x x}$ and $p_{x y}$ are the stresses (second-order moment) and $\boldsymbol{q}$ is related to the heat flux (third-order moments), the transform matrix $\mathrm{M}$ is given by [18]:

$$
\mathbf{M}=\left(\begin{array}{ccccccccc}
1 & 1 & 1 & 1 & 1 & 1 & 1 & 1 & 1 \\
-4 & -1 & -1 & -1 & -1 & 2 & 2 & 2 & 2 \\
4 & -2 & -2 & -2 & -2 & 1 & 1 & 1 & 1 \\
0 & 1 & 0 & -1 & 0 & 1 & -1 & -1 & 1 \\
0 & -2 & 0 & 2 & 0 & 1 & -1 & -1 & 1 \\
0 & 0 & 1 & 0 & -1 & 1 & 1 & -1 & -1 \\
0 & 0 & -2 & 0 & 2 & 1 & 1 & -1 & -1 \\
0 & 1 & -1 & 1 & -1 & 0 & 0 & 0 & 0 \\
0 & 0 & 0 & 0 & 0 & 1 & -1 & 1 & -1
\end{array}\right)
$$

The matrix $\mathrm{M}$ maps the distribution functions to its moments:

$$
\mathbf{m}=\mathbf{M} \cdot \mathbf{f}, \quad \mathbf{f}=\mathrm{M}^{-1} \cdot \mathbf{m} .
$$

The labeling of the discrete velocity set $\left\{\boldsymbol{c}_{i}\right\}$ is uniquely defined by the rows 4 and 6 in $\mathrm{M}$ corresponding to $c_{i x}$ and $c_{i y}$, respectively. For the construction of 
$\mathrm{M}$ and detailed description of the moments, we refer readers to the work by d'Humières et al. [18,20].

The diagonal matrix $\hat{\mathrm{S}}$ of relaxation rates $\left\{s_{i}\right\}$ is given by:

$$
\hat{\mathrm{S}}=\operatorname{diag}\left(0, s_{2}, s_{3}, 0, s_{5}, 0, s_{7}, s_{8}, s_{9}\right)
$$

where the relaxation rates $s_{8}=s_{9}=s_{\nu}=1 / \tau$ determines the dimensionless viscosity of the model:

$$
\nu=\frac{1}{3}\left(\tau-\frac{1}{2}\right) c \delta_{x}
$$

where $c:=\delta_{x} / \delta_{t}$. Since the parameter $\tau$ is dimensionless, then the physical viscosity is proportional to a scaling factor, depending on the adopted grid size and time step (diffusion scaling is assumed). Other relaxation rates $s_{2}, s_{3}$ and $s_{5}=s_{7}=s_{q}$ are usually determined by linear stability of the model [18]. In addition, the no-slip boundary conditions will also determine the choice of $s_{5}=s_{7}[7,8]$.

In Eq. (1), the equilibria for the non-conserved moments for the D2Q9 model are:

$$
\begin{aligned}
& e^{(\mathrm{eq})}=-2 \delta \rho+3 \boldsymbol{j} \cdot \boldsymbol{j}, \quad \varepsilon^{(\mathrm{eq})}=\delta \rho-3 \boldsymbol{j} \cdot \boldsymbol{j}, \\
& q_{x}^{(\mathrm{eq})}=-j_{x}, \quad q_{y}^{(\mathrm{eq})}=-j_{y}, \\
& p_{x x}^{(\mathrm{eq})}=j_{x}^{2}-j_{y}^{2}, \quad p_{x y}^{(\mathrm{eq})}=j_{x} j_{y} .
\end{aligned}
$$

where $\delta \rho$ is the density fluctuation, $\rho=\bar{\rho}+\delta \rho$ and $\bar{\rho}=1, \boldsymbol{j}:=\left(j_{x}, j_{y}\right)=$ $\left(\bar{\rho} u_{x}, \bar{\rho} u_{y}\right)=\left(u_{x}, u_{y}\right)$ is the flow momentum. Here the approximation for incompressible flows has been used [41], i.e., the coupling between the density fluctuation $\delta \rho$ and the flow velocity $\boldsymbol{u}$ is neglected in $\mathbf{m}^{(\mathrm{eq})}$. Note that the equilibria of the conserved moments ( $\rho$ and $\boldsymbol{j}:=\rho \boldsymbol{u}$ ) are equal to the conserved moments themselves. The above equilibria $\mathbf{m}^{(\mathrm{eq})}$ are equivalent to the following equilibrium distribution functions:

$$
f_{i}^{(\mathrm{eq})}=w_{i}\left\{\delta \rho+\frac{\left(\boldsymbol{c}_{i} \cdot \boldsymbol{u}\right)}{R T}+\frac{1}{2}\left[\frac{\left(\boldsymbol{c}_{i} \cdot \boldsymbol{u}\right)^{2}}{(R T)^{2}}-\frac{\boldsymbol{u} \cdot \boldsymbol{u}}{R T}\right]\right\}
$$

where $T$ is the temperature of the flow (assumed constant), $R$ is the specific gas constant and $R T$ represents the internal energy of the fluid, which is a constant for isothermal flows considered here. In the LBE model we use here, $R T=c^{2} / 3[2,3,18]$. For the D2Q9 model, the coefficients $w_{0}=4 / 9, w_{i}=1 / 9$ for $\left\|\boldsymbol{c}_{i}\right\|=1, i=1-4$, and $w_{i}=1 / 36$ for $\left\|\boldsymbol{c}_{i}\right\|=\sqrt{2}, i=5-8$. 


\subsection{No-slip boundary conditions for curved boundaries}

We will use the interpolated bounce-back (IBB) boundary conditions (BCs) to model the no-slip fluid-solid boundary conditions with curved boundaries [29]. The bounce-back boundary conditions are based on the intuitive picture that a particle reverses its momentum when colliding with a solid wall at the rest. If the wall is moving with a certain velocity $\boldsymbol{u}_{\mathrm{w}}$, a particle colliding with the wall should also gain additional momentum from the wall. Based on this intuitive picture, as illustrated in Fig. 1, if the wall is located one half grid spacing beyond the last node $\boldsymbol{r}_{\mathrm{A}}$ in flow domain, then a particle with velocity $\boldsymbol{c}_{1}$ at node $\boldsymbol{r}_{\mathrm{A}}$ and time $t_{n}$ collides with the wall at $\boldsymbol{r}_{\mathrm{w}}$, reverses its momentum, and returns to $\boldsymbol{r}_{\mathrm{A}}$. Therefore, when the boundary location is not precisely located at $\delta_{x} / 2$ beyond the last flow node, interpolations or other means must be used to reconstruct the distribution functions at the desirable nodes in flow domain.
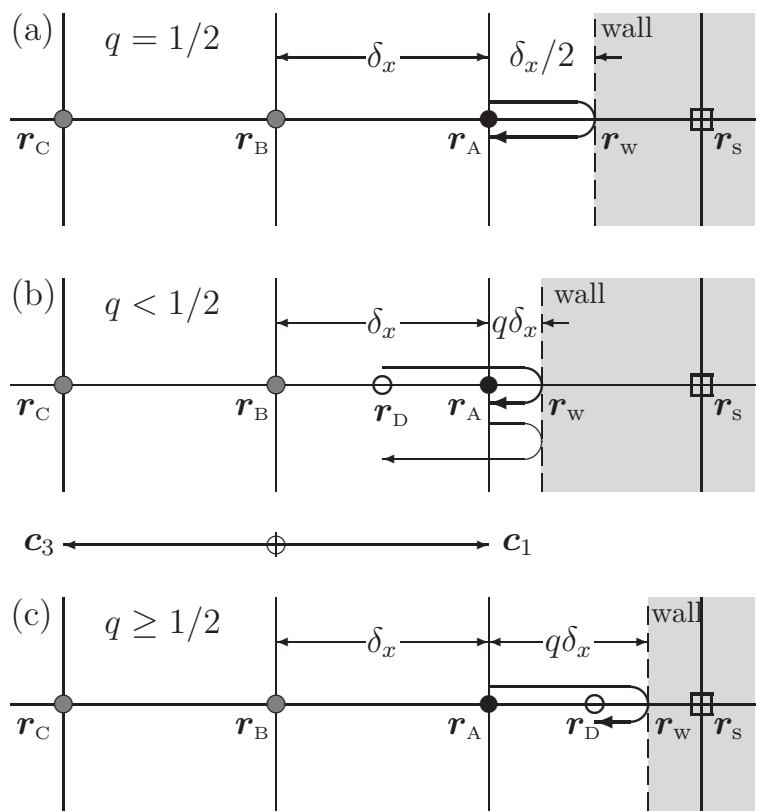

Fig. 1. Illustration of the bounce-back (BB) boundary conditions (BCs). (a) $q=1 / 2$, the "perfect" BB-BCs without interpolation. (b) $q<1 / 2$, the BB-BCs with interpolations before the collision with the wall located at $\boldsymbol{r}_{\mathrm{w}}$. (c) $q \geq 1 / 2$, the BB-BCs with interpolations after the collision with the wall.

The flow nodes adjacent to a solid boundary have links connecting these nodes to their neighboring solid or boundary nodes beyond the flow domain. These links intersect with the boundary so that part of them lie inside the flow domain and part of them are outside, as illustrated by the link between $\boldsymbol{r}_{\mathrm{A}}$ and $\boldsymbol{r}_{\mathrm{s}}$ in Fig. 1. Define the parameter $q$ as the fraction of a link between a 
flow node and a boundary node which lies in flow domain as

$$
q:=\frac{\left\|\boldsymbol{r}_{\mathrm{A}}-\boldsymbol{r}_{\mathrm{w}}\right\|}{\left\|\boldsymbol{r}_{\mathrm{A}}-\boldsymbol{r}_{\mathrm{s}}\right\|},
$$

as depicted in Fig. 1, then one has to treat the following two scenarios separately:

- When $q<\frac{1}{2}, f_{i}\left(\boldsymbol{r}_{\mathrm{D}}\right)$ can be constructed from the $f_{i}$ 's in the nearby nodes before the bounce-back collision, so that $f_{\bar{\imath}}\left(\boldsymbol{r}_{\mathrm{A}}\right)$ is obtained after the bounceback collision with the wall located at $\boldsymbol{r}_{\mathrm{w}}$;

- When $q \geq \frac{1}{2}, f_{\bar{\imath}}\left(\boldsymbol{r}_{\mathrm{A}}\right)$ is obtained with $f_{\bar{\imath}}\left(\boldsymbol{r}_{\mathrm{D}}\right)$ after the bounce-back collision and $f_{\bar{\imath}}$ at other nearby nodes;

where $\boldsymbol{c}_{\bar{\imath}}:=-\boldsymbol{c}_{i}$ is assumed to be the bounced back particle velocity.

To reconstruct the distribution functions $f_{\bar{\imath}}\left(\boldsymbol{r}_{\mathrm{A}}\right)$ entering the flow domain from boundary nodes, we can use either the linear interpolations:

$$
\begin{aligned}
& f_{\bar{\imath}}\left(\boldsymbol{r}_{\mathrm{A}}, t_{n+1}\right)=(1-2 q) f_{i}\left(\boldsymbol{r}_{\mathrm{A}}, t_{n+1}\right)+2 q f_{i}^{*}\left(\boldsymbol{r}_{\mathrm{A}}, t_{n}\right), \quad q<1 / 2, \\
& f_{\bar{\imath}}\left(\boldsymbol{r}_{\mathrm{A}}, t_{n+1}\right)=\frac{(2 q-1)}{2 q} f_{\bar{\imath}}^{*}\left(\boldsymbol{r}_{\mathrm{A}}, t_{n}\right)+\frac{1}{2 q} f_{i}^{*}\left(\boldsymbol{r}_{\mathrm{A}}, t_{n}\right), \quad q \geq 1 / 2,
\end{aligned}
$$

or the quadratic interpolations:

$$
\begin{aligned}
f_{\bar{\imath}}\left(\boldsymbol{r}_{\mathrm{A}}, t_{n}+\delta_{t}\right)= & q(1+2 q) f_{i}^{*}\left(\boldsymbol{r}_{\mathrm{A}}, t_{n}\right)+\left(1-4 q^{2}\right) f_{i}^{*}\left(\boldsymbol{r}_{\mathrm{A}}-\boldsymbol{c}_{i} \delta_{t}, t_{n}\right) \\
& +q(2 q-1) f_{i}^{*}\left(\boldsymbol{r}_{\mathrm{A}}-2 \boldsymbol{c}_{i} \delta_{t}, t_{n}\right), \quad 0<q<1 / 2, \\
f_{\bar{\imath}}\left(\boldsymbol{r}_{\mathrm{A}}, t_{n}+\delta_{t}\right)= & \frac{1}{q(1+2 q)} f_{i}^{*}\left(\boldsymbol{r}_{\mathrm{A}}, t_{n}\right)+\frac{(2 q-1)}{q} f_{\bar{\imath}}^{*}\left(\boldsymbol{r}_{\mathrm{A}}, t_{n}\right) \\
& +\frac{(1-2 q)}{(1+2 q)} f_{\bar{\imath}}^{*}\left(\boldsymbol{r}_{\mathrm{A}}-\boldsymbol{c}_{i} \delta_{t}, t_{n}\right), \quad 1 / 2 \leq q<1,
\end{aligned}
$$

where $f_{i}^{*}$ denotes the post-collision distribution function. It must be stressed that $q=0$ and $q=1$ are singular cases. When $q=1 / 2$, both the linear and quadratic interpolated bounce-back boundary conditions reduce to the original bounce-back boundary conditions:

$$
f_{\bar{\imath}}\left(\boldsymbol{r}_{\mathrm{A}}, t_{n+1}\right)=f_{i}^{*}\left(\boldsymbol{r}_{\mathrm{A}}, t_{n}\right) .
$$

While the bounce-back boundary conditions are the most often used, they are also misunderstood or misinterpreted very often. By no means the intuitive picture illustrated in Fig. 1 should be taken literally. The precise location where the no-slip boundary conditions are satisfied is model dependent. For the incompressible Poiseuille flow with its boundaries parallel to a lattice axis, 
it can be shown analytically that the no-slip boundary location is precisely one half lattice spacing beyond the last flow node if and only if the following relation is satisfied $[5,7]$ :

$$
s_{q}=8 \frac{\left(2-s_{\nu}\right)}{\left(8-s_{\nu}\right)}
$$

where $s_{\nu}=s_{8}=s_{9}=1 / \tau$ is the relaxation rate for $p_{x x}$ and $p_{x y}$ which also determines the shear viscosity $\nu$ given by Eq. (5) and $s_{q}=s_{5}=s_{7}$ is the relaxation rate for $\boldsymbol{q}=\left(q_{x}, q_{y}\right)$. Obviously, such a relationship cannot be satisfied by the lattice BGK equation with the single-relaxation-time collision model, therefore the no-slip boundary location depends on the relaxation parameter $\tau$ in lattice BGK models $[7,42,8]$.

\subsection{Inflow and outflow boundary conditions}

The inflow and outflow boundary conditions used in the LBE simulations are either the Dirichlet or Neumann types for the hydrodynamic variables $p$ (or $\rho$ ) and $\boldsymbol{u}$. In an athermal LBE model of $Q$ velocities in $d$ dimensions, we have $(d+1)$ conserved variables, i.e., $\rho$ and $\rho \boldsymbol{u}$, among all $Q$ moments. The hydrodynamic boundary conditions for $\rho$ and $\rho \boldsymbol{u}$ do not specify the boundary conditions for the remaining $(Q-d)$ non-conserved or kinetic moments, which are important in the LBE. We will discuss how to consistently treat these kinetic moments in the boundary conditions.

The Dirichlet boundary conditions for either velocity $\boldsymbol{u}$ or pressure $p$ can be realized by the procedures described bellow. Assume a 2D computational domain is covered by a rectangular uniform mesh without stretching, with nodes labeled by index $(i, j), i \in\left\{1,2, \ldots, N_{x}\right\}$ and $j \in\left\{1,2, \ldots, N_{y}\right\}$, and the streamwise direction is along the $x$-axis. As an example, we will use the inlet velocity boundary conditions at the left of the mesh to illustrate the proposed boundary conditions. The velocity $\boldsymbol{u}(i=1, j)=\boldsymbol{u}_{\text {in }}(j)$ is imposed at the inlet $i=1$. While the velocity $\boldsymbol{u}(i=1, j)=\boldsymbol{u}_{\text {in }}(j)$ at $i=1$ remained intact, all other moments are copied from the line $i=2$ adjacent to the inlet. These moments are then transformed to the distribution functions $\mathrm{f}(i=1, j)$, which are used as the boundary conditions at the inlet. Thus the velocity boundary condition at the inlet $i=1$ can be written as

$$
\mathbf{f}(i=1, j)=\left.\mathbf{M}^{-1} \cdot \mathbf{m}^{*}(i=2, j)\right|_{\boldsymbol{u}=\boldsymbol{u}_{\text {in }}},
$$

where $\mathbf{m}^{*}$ denotes the post-collision moments. Similarly, the pressure boundary condition $p(i=1, j)=p_{\text {in }}(j)$ can be realized as

$$
\mathbf{f}(i=1, j)=\left.\mathbf{M}^{-1} \cdot \mathbf{m}^{*}(i=2, j)\right|_{\rho=\rho_{\text {in }}},
$$

where $\rho_{\text {in }}=p_{\text {in }} / c_{s}^{2}$. In the proposed boundary boundary conditions described 
above, all the non-equilibrium moments are generated in the flow domain through the evolution process consistent with the flow, only the hydrodynamic variables, either $\boldsymbol{u}$ or $\rho$, relevant to specific boundary conditions are imposed at the boundary. Therefore, as the flow develops, non-equilibrium (non-conserved) moments and the equilibrium moments which are not imposed by given boundary conditions at the boundary are expected to consistently evolve according to flow dynamics. In the proposed boundary conditions, no interpolations or extrapolations are needed. For simulations in this work, we usually use the velocity boundary conditions at the inlet, and the constant pressure boundary condition at the outlet:

$$
\rho_{\text {out }}=\text { constant. }
$$

We will compare the proposed boundary conditions described above with the non-equilibrium bounce-back boundary conditions [14], in which the velocity boundary conditions $\boldsymbol{u}(i=1, j)=\boldsymbol{u}_{\text {in }}(j)$ at the inlet is realized as:

$$
\begin{aligned}
f_{\bar{k}}(i=1, j) & =f_{k}(i=2, j)-f_{k}^{(\mathrm{eq})}(i=2, j)+\left.f_{\bar{k}}^{(\mathrm{eq})}(i=2, j)\right|_{\boldsymbol{u}=\boldsymbol{u}_{\text {in }}(j)} \\
& =f_{k}(i=2, j)-3 w_{k} \boldsymbol{c}_{k} \cdot\left[\boldsymbol{u}(i=2, j)+\boldsymbol{u}_{\text {in }}(j)\right]
\end{aligned}
$$

where $f_{\bar{k}}$ is the distribution function corresponding to the discrete velocity $\boldsymbol{c}_{\bar{k}}$, and $\boldsymbol{c}_{\bar{k}}:=-\boldsymbol{c}_{k}$ is an incoming discrete particle velocity with respect to the boundary at $i=1$, i.e., those velocities with positive $x$ component $\left(c_{\bar{k} x}>0\right)$ hence $\left\{f_{\bar{k}}(i=1, j)\right\}$ are the incoming distribution functions. In the above derivation, we have assumed the incompressible LBE model [41] with the equilibria of Eq. (7). Similarly, when the pressure boundary condition $p(i=$ $1, j)=p_{\text {in }}(j)$ is imposed at the entrance, or equivalently $\rho(i=1, j)=\rho_{\text {in }}(j)$ through $\rho=p / c_{s}^{2}$, the non-equilibrium bounce-back boundary conditions are

$$
f_{\bar{k}}(i=1, j)=f_{k}(i=2, j)-w_{k}\left[\rho(i=2, j)-\rho_{\text {in }}(j)\right]-6 w_{k} \boldsymbol{c}_{k} \cdot \boldsymbol{u}(i=2, j) .
$$

Another way of implementing a constant pressure $p=p_{\text {out }}\left(\rho=\rho_{\text {out }}=p_{\text {out }} / c_{s}^{2}\right)$ at the outlet $i=N_{x}$ is to impose equilibrium distributions:

$$
f_{k}\left(i=N_{x}, j\right)=f_{k}^{(\mathrm{eq})}\left(\rho=\rho_{\text {out }}, \boldsymbol{u}=\boldsymbol{u}\left(i=N_{x}-1, j\right)\right) .
$$

With the MRT-LBE, the inflow and outflow boundary conditions discussed above are particularly easy to implement. After advection and collision, the moments at $i=2$ are copy to $i=1$, then either the velocity $\boldsymbol{u}$ or the density $\rho$ are reset according to given boundary conditions, while the rest of the moments are remained intact. The moments $\left\{m_{i}\right\}$ are transformed back to the distribution functions $\left\{f_{i}\right\}$, which carry the information imposed by the boundary conditions to flow domain. This differs from the approach that sets 
the distribution functions $\left\{f_{i}\right\}$ to their equilibria $\left\{f_{i}^{(\mathrm{eq})}\right\}$ with the specified values of hydrodynamic variables at the boundaries [9] or that uses extrapolations to obtain non-equilibrium distribution functions $[10,13]$. The proposed approach will be tested numerically in Section 3.1.

\subsection{Force evaluation at flow-solid boundaries}

Two methods to compute hydrodynamic forces on a flow-solid boundary in the lattice Boltzmann simulations are used. The first method is to compute the pressure and stresses at the flow-solid boundary and then to integrate the forces over the entire boundary. For arbitrary curved boundaries, the hydrodynamic forces at boundary can be obtained by interpolating either the pressure and velocity fields from flow nodes to boundary locations [30,31,43-45], or the distribution functions to compute the local stresses.

The second method is the momentum exchange algorithm $[46,43]$ which is only applicable to the LBE method. The momentum exchange algorithm is directly related to the bounce-back boundary conditions: for a particle distribution function $f_{i}^{*}\left(\boldsymbol{r}_{A}, t_{n}\right)$ at a flow node $\boldsymbol{r}_{A}$ adjacent to a boundary node $\boldsymbol{r}_{\mathrm{s}}$ is bounced back as $f_{\bar{\imath}}\left(\boldsymbol{r}_{A}, t_{n+1}\right)$ after colliding with the wall, as illustrated in Fig. 1. Consequently the hydrodynamic force $\delta \boldsymbol{F}$ due to this flow-wall interaction is:

$$
\delta \boldsymbol{F}\left(\boldsymbol{r}_{\mathrm{w}}, t_{n+1 / 2}\right)=\left[f_{\bar{\imath}}\left(\boldsymbol{r}_{A}, t_{n+1}\right)+f_{i}^{*}\left(\boldsymbol{r}_{A}, t_{n}\right)\right] \boldsymbol{c}_{i},
$$

where $\boldsymbol{c}_{i}$ points from a flow node to a boundary node. For a body of volume $\Omega$ and boundary $\partial \Omega$, let $\mathcal{B}\left(\boldsymbol{r}_{k}\right)$ be the set of the flow notes $\left\{\boldsymbol{r}_{k}\right\}$ next to $\partial \Omega$ which have at least one link $\boldsymbol{c}_{i}$ intersecting with $\partial \Omega$. The the total hydrodynamic force on the body is simply given by:

$$
\boldsymbol{F}=\sum_{\boldsymbol{r}_{k} \in \mathcal{B}} \sum_{\boldsymbol{c}_{i} \cap \partial V \neq 0}\left[f_{\bar{\imath}}\left(\boldsymbol{r}_{\mathrm{b}}, t_{n+1}\right)+f_{i}^{*}\left(\boldsymbol{r}_{\mathrm{b}}, t_{n}\right)\right] \boldsymbol{c}_{i} .
$$

The momentum exchange algorithm is simple to implement, its accuracy and efficiency have been validated previously $[46,43,47]$. Therefore it is the method to be used in the present work.

\subsection{Grid stretching and interpolated $L B E$}

The lattice Boltzmann equation employs a uniform Cartesian mesh with the grid spacing $\delta_{x}$. To use non-uniform mesh, one can use either local grid refinement $[15,25,26]$ or grid stretching $[27,2,28]$ and the latter approach is used in the present work. The fundamental difference between the grid refinement and grid stretching methods is the following. In the grid refinement, the grid 
spacing $\delta_{x}$ and the time step size $\delta_{t}$ are refined consistently, hence the viscosity $\nu$ must be rescaled accordingly to maintain the Reynolds and Mach numbers fixed throughout the system $[15,25,26]$, interpolations are used to compute data only at the interface between two meshes of different grid spacing $\delta_{t}$. In the grid stretching approach, the time step size $\delta_{t}$ remains the same regardless of the grid spacing $\Delta x$, and $\delta_{x}$ is the grid spacing with which the advection process can transfer data from one grid node to its neighboring ones. In this approach, one can use an arbitrary non-uniform mesh with the finest grid spacing $\Delta x=\delta_{x}$. For the nodes with $\Delta x>\delta_{x}$, interpolations must be use to compute the values of $\left\{f_{i}\right\}$ on these grid nodes after advection. Because $\delta_{t}$ and $\delta_{x}$ remain the same in the grid stretching approach, the viscosity remains intact throughout the system.

In this work we will use a very simple grid stretching strategy for a two dimensional Cartesian mesh. Surrounding a rectangular uniform fine mesh with grid spacing $\delta_{x}$, the grid spacings are stretched exponentially along both $x$ and $y$ directions beyond the four boundaries of the fine mesh, as illustrated by Fig. 2. The stretched grids are given by:

$$
\begin{aligned}
& \Delta x_{k}:=x_{k}-x_{0}=\delta_{x} \exp \left(\Delta x_{k-1} / D\right), \\
& \Delta y_{k}:=y_{k}-y_{0}=\delta_{x} \exp \left(\Delta y_{k-1} / D\right),
\end{aligned}
$$

where $D$ is a characteristic length, index $k$ is only used for the stretched grids, and $x_{0}$ and $y_{0}$ are the coordinates of the boundaries of the fine mesh from which the grid spacings are stretched, and $\delta_{x}=1=\Delta x_{0}$.

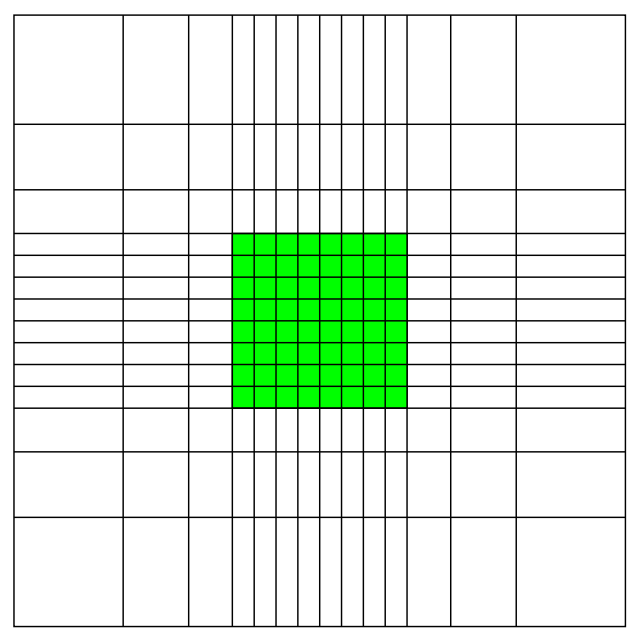

Fig. 2. Illustration of stretched mesh. The shaded part is the fine mesh of the grid space $\delta_{x}=1$.

With non-uniform Cartesian meshes, interpolations must be used in order to obtain the values of the distribution functions $\left\{f_{i}\right\}$, because advection transfers the data off the grid nodes. In the grid stretching approach, we apply interpolations after the collision step to compute the values of $\left\{f_{i}\right\}$ on a grid 


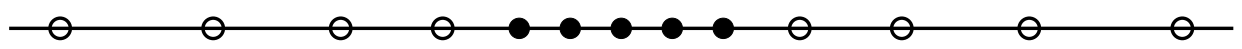

Fig. 3. Illustration of stretched mesh in one dimension. The grid spacing between two adjacent disks is $\Delta x=\delta_{x}=1$, and that between two circles or a circle and a disk is $\Delta x>\delta_{x}=1$. For nodes indicated by circles, interpolations are necessary to compute the data $\left\{f_{i}\right\}$ after advection.

node from the nearby off-grid values of $\left\{f_{i}\right\}$, as illustrated in Fig. 3. The advection moves $\left\{f_{i}\right\}$ from one grid node to the next when $\Delta x=\delta_{x}=1$, as indicated by the disks in Fig. 3. However, when $\Delta x>\delta_{x}=1$, as indicated by the circles in Fig. 3, the advection moves $\left\{f_{i}\right\}$ to off-grid locations. Therefore interpolations must be used to reconstruct $\left\{f_{i}\right\}$ on the grid nodes indicated by circles. For those $f_{i}$ 's moving along lattice lines, i.e., along $x$ - and $y$-axis, we use second order interpolations involving three points along a grid line. For those $f_{i}$ 's moving along diagonal directions, we apply second order interpolations in both $x$ - and $y$-direction, which involves nine points $[27,28,48,49,47]$.

Clearly, interpolations introduce numerical dissipation. However, so long as the interpolations are second or higher order, they do not affect the formal order of accuracy $[27,28]$. Although interpolations can introduce severe dissipation in small scales [50], they can be judicially used to enhance computational efficiency without degrading numerical results $[27,28,48,49,47]$.

\section{Validation of the numerical method}

In this section we validate our proposed approach to realize Dirichlet boundary conditions and non-uniform mesh with stretched grids. All the validations are carried out in two dimensional flows. We first compare our proposed boundary conditions with the non-equilibrium bounce-back scheme for the Poiseuille flow and the results are presented in Section 3.1. Our second validation test for the boundary conditions is the flow past a cylinder asymmetrically placed in a channel with the Reynolds number $R e=20$ and 100, corresponding to steady and unsteady flow, respectively. A uniform mesh is used for this flows and the results are given in Section 3.2. Our last validation test is the flow past a cylinder in an unbounded domain with $\mathrm{Re}=100$. In this case the nonuniform mesh with stretched grids has to be used. The results are presented in Section 3.3.

\subsection{Poiseuille flow}

To validate of the inflow/outflow boundary conditions implemented with the

MRT-LBE, we consider first the steady Poiseuille flow in two-dimensions [51], 
for which the incompressible Navier-Stokes equation admits an analytic solution for the streamwise velocity $u(y)$. In our simulations, the streamwise direction is along the $x$-axis and the spanwise direction is along the $y$-axis. The computational domain is $(x, y) \in[0, L] \times[0, H]=\Omega$, where $L$ and $H$ are channel length and height, respectively. With constant pressure $p_{0}$ and $p_{1} \mathrm{im}$ posed at the inlet and outlet, respectively, the pressure $p$ and the streamwise velocity $u(y)$ have the following solutions:

$$
\begin{aligned}
& p(x)=p_{0}-\frac{\left(p_{0}-p_{1}\right) x}{L}, \\
& u(y)=U_{\max }\left[1-\left(\frac{2 y}{H}\right)^{2}\right], \quad-\frac{H}{2} \leq y \leq \frac{H}{2},
\end{aligned}
$$

where the maximum streamwise velocity along the channel center line is a constant:

$$
U_{\max }=\frac{H^{2}\left(p_{0}-p_{1}\right)}{8 L \rho \nu} .
$$

For near incompressible flow, we can assume $\rho=1$ in $U_{\max }$.

To validate the consistency of the proposed boundary conditions, we impose a parabolic velocity profile corresponding to $p_{0}$ at the inlet $x / L=0$ and constant pressure boundary condition at the outlet $x / L=1$, as given be Eqs. (12) and (13), respectively. At the walls, bounce-back boundary conditions are applied. The system size used in our test is $N_{x} \times N_{y}=20 \times 21$. The initial velocity field is set to be zero every where. The values of the relaxation rates are: $s_{2}=1.63$, $s_{3}=1.14, s_{q}=s_{5}=s_{7}=1.92$ and $s_{\nu}=s_{8}=s_{9}=1 / \tau$. These values of $s_{2}, s_{3}$ and $s_{5}=s_{7}$ will be used throughout this study unless otherwise stated. We vary the values of $\tau$ and $p_{1}$ in such a way that $U_{\max }=0.1 \mathrm{c}$ is fixed in our test. The value of $s_{\nu}=1 / \tau$ used in our test are: 1.0, 1.3, 1.6, 1.85, 1.9, 1.95, 1.98 and 1.99 , i.e., $\nu \in\left[8.375 \cdot 10^{-4}, 1 / 6\right]$. We compare our proposed boundary conditions of Eqs. (12) and (13) with the nonequilibrium bounce-back (NEQ$\mathrm{BB})$ boundary conditions at the inlet and the equilibrium boundary conditions at the outlet, given by Eqs. (14) and (16), respectively.

When the system reaches steady state, attained after about 3,000 iterations for the smallest viscosity $\nu \approx 8.375 \cdot 10^{-4}$, we measure the velocity along the channel center and the result is shown in Fig. 4(a). Clearly, the proposed boundary conditions are more accurate than the nonequilibrium bounce-back boundary conditions: the velocity $U_{\max }$ obtained with the proposed boundary conditions varies at the $10^{-6}$ digit, while that obtained with the NEQ-BB boundary conditions is three order of magnitude larger, it varies at the $10^{-3}$ digit.

We next quantify the error in the measured viscosity $\nu^{*}$ in the same tests with a fixed inflow velocity profile of $U_{\max }=0.1 c$ and varying $\tau$ and $p_{1}$. We measure 

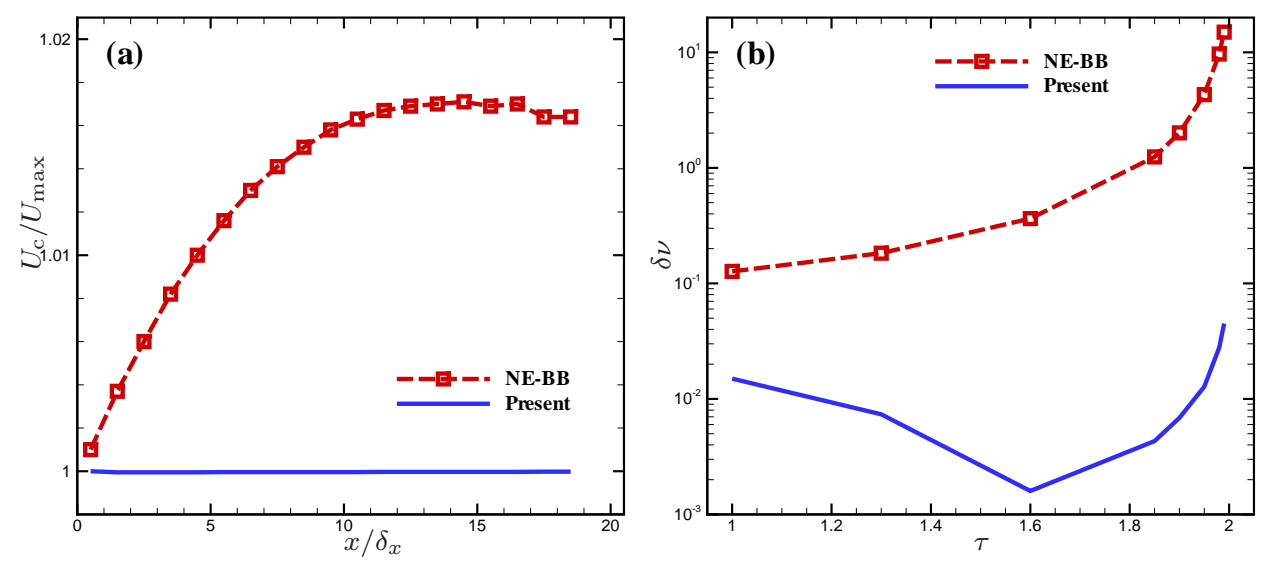

Fig. 4. 2D Poiseuille flow with different implementations of the inflow and out boundary conditions. (a) The normalized streamwise velocity along the channel center $U_{\mathrm{c}} / U_{\max }$, with a fixed $\tau=1 / 1.85$. (b) The $\tau$-dependence of the maximum relative error $\delta \nu$ given by Eq. (21) for $\tau=1 / s_{\nu} \in[1,1.99]$.

the relative error in $\nu$ :

$$
\delta \nu=\frac{\left|\nu^{*}-\nu\right|}{\nu}
$$

where $\nu$ is computed from Eq. (20), and $\nu^{*}$ is measured from the numerical simulations with varying $\tau$ and $p_{1}$. The results for $\delta \nu$ are showed in Fig. 4(b). In the range of the relaxation rate $s_{\nu}=1 / \tau \in[1,1.99]$, the error in $\nu$ in the simulations with the NEQ-BB boundary conditions is much larger than that with the proposed boundary conditions, and the difference is particularly apparent when $\tau$ is close to 2. This simple test clear shows the advantages of the proposed boundary conditions.

\subsection{Flow past a cylinder in a channel}

Our second test case to validate our code is the flow past a cylinder asymmetrically placed in channel in 2D [32]. This flow has been used as a benchmark test [32], thus we can compare our results with existing data. The flow configuration is illustrated in Fig. 5. We use an uniform mesh of size $N_{x} \times N_{y}$ for the simulations presented in this section. At the inlet, a parabolic velocity profile with maximum velocity $U_{\max }$ is imposed. At the outlet, a constant pressure boundary condition corresponding to $\rho_{\infty}=1$ is used. At the channel walls, the bounce-back boundary conditions are applied. At the cylinder boundary, we use the bounce-back, the first-order and second-order interpolated bounce-back boundary conditions [16,8]. The Reynolds number is defined by the average inflow velocity $\bar{U}=2 U_{\max } / 3$ and the cylinder diameter $D$, i.e., $\operatorname{Re}=\bar{U} D / \nu=2 U_{\max } D / 3 \nu$.

At $\operatorname{Re}=20$, the flow is steady and a recirculation bubble is formed behind the cylinder. The quantities measured are the drag coefficient $C_{D}$, the lift coeffi- 


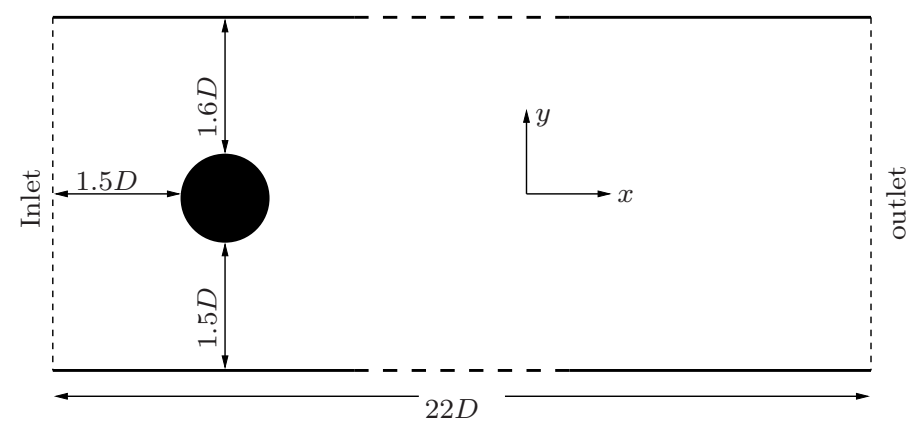

Fig. 5. The geometric configuration for the flow past a cylinder asymmetrically placed in the channel.

cient $C_{L}$, the recirculation bubble length $L_{r}$, and $\Delta \bar{p}$, the pressure difference between the front and the back of the cylinder normalized by $\rho_{\infty} \bar{U}^{2} / 2$. We use a number of meshes with different resolutions in terms of $D / \delta_{x}$ and our results are summarized in Table 1.

Table 1

Flow past a cylinder asymmetrically placed in a channel at $\operatorname{Re}=20$. The meshsize dependence of the drag coefficient $C_{D}$, the lift coefficient $C_{L}$ and the length of the recirculating zone $L_{r} / D$. BB, I and II denote the bounce-back, and the firstorder and second-order interpolated bounce-back boundary conditions. The results of Ref. [32] are also included.

\begin{tabular}{c|c|ccccc|c}
\hline \hline & $D / \delta_{x}$ & 10 & 20 & 30 & 40 & 80 & NS [32] \\
\hline \hline \multirow{4}{*}{$C_{D}$} & BB & 6.171 & 5.816 & 5.755 & 5.6855 & 5.6108 & \\
& I & 5.6627 & 5.5741 & 5.5631 & 5.5600 & 5.5591 & $5.5700-5.5900$ \\
& II & 5.6306 & 5.5621 & 5.5574 & 5.5573 & 5.5584 & \\
\hline \multirow{3}{*}{$C_{L}$} & BB & 0.0354 & 0.0223 & 0.0186 & 0.0164 & 0.0129 & \\
& I & 0.0158 & 0.0123 & 0.0117 & 0.0115 & 0.0113 & $0.0104-0.0110$ \\
& II & 0.0170 & 0.0134 & 0.0125 & 0.0116 & 0.0113 & \\
\hline \multirow{3}{*}{$L_{r} / D$} & BB & 0.8953 & 0.8619 & 0.8629 & 0.8531 & 0.8480 & \\
& I & 0.7696 & 0.8237 & 0.8322 & 0.8358 & 0.8402 & $0.8420-0.8520$ \\
& II & 0.7728 & 0.8259 & 0.8344 & 0.8371 & 0.8402 & \\
\hline & BB & 6.1549 & 5.7454 & 5.7500 & 5.7856 & 5.8132 & \\
$\Delta \bar{p}$ & I & 5.6975 & 5.8305 & 5.7636 & 5.7823 & 5.8132 & $5.8600-5.8800$ \\
& II & 5.7947 & 5.8470 & 5.7762 & 5.7836 & 5.8091 & \\
\hline \hline
\end{tabular}

Figure 6 shows the contours of the vorticity $\omega$ at the inlet and outlet sections of the channel. The maximum value of $|\omega|$ is about 1.0 and it is generated within a very thin layer around the cylinder. Away from the cylinder, the vorticity is rather weak. At the four corners of of the channel, the magnitude 
of $\omega$ is about $10^{-3}$ at the inlet and about $10^{-4}$ at the outlet, indicating that the proposed inlet/outlet boundary conditions do not generate spurious effects near the corners.
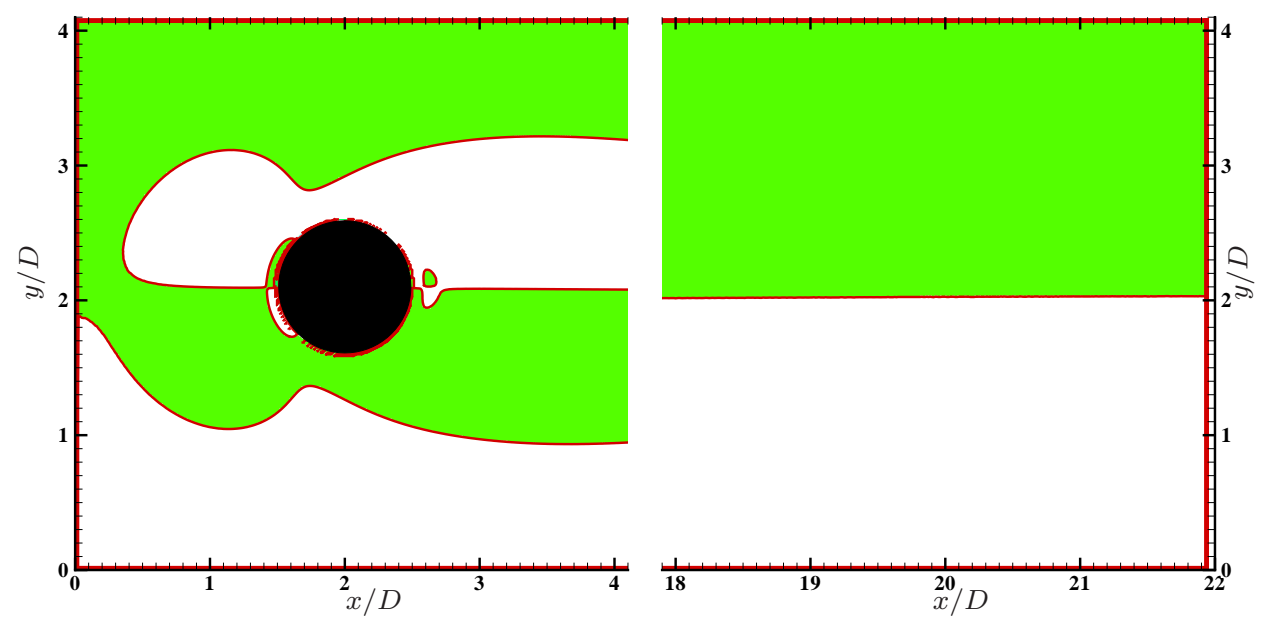

Fig. 6. The vorticity contours for the flow past a cylinder asymmetrically placed in the channel at $\operatorname{Re}=20$, with the resolution $D / \delta_{x}=80$.

Figure 7 shows the pressure coefficient $C_{p}$ around the cylinder:

$$
C_{p}(\theta)=\frac{p(\theta)-p_{\infty}}{\frac{1}{2} \rho_{\infty} U_{\max }^{2}},
$$

where $\rho_{\infty}=1, p_{\infty}=1 / 3, U_{\max }=0.1 c$, and $\theta=0^{\circ}$ is the stagnation point in front of the cylinder. In order to compute the pressure coefficient $C_{p}$, the pressure $p(\theta)$ at the cylinder surface is extrapolated from mesh points in the flow domain. With the resolution $D / \delta_{x}=40, C_{p}(\theta)$ computed with the bounceback boundary conditions around the cylinder shows considerable oscillations, while the results obtained with the first- and second-order interpolations are much smoother, as expected. We also note that the results obtained by the first-order and second-order interpolations are very close to each other, i.e., the second-order interpolations do not significantly improve the result of $C_{p}(\theta)$.

At $\operatorname{Re}=100$, the flow becomes unsteady and a periodic vortex shedding takes place. Consequently both the drag and lift coefficients are periodic functions in time. We measure the Strouhal number St, the maximum drag coefficient $C_{D}^{\max }$ and the maximum lift coefficient $C_{L}^{\max }$ with different grid resolutions indicated by $D / \delta_{x}$. Our results are summarized in Table 2 .

It is worth the effort to point out that BB boundary condition produces a very good result for $D / \delta_{x}=10$. This is not systematic: in fact, BB shows only a first order error decay for $D / \delta_{x}>10$, while I and II interpolations show better trends. In a convergence study, it may happen that the target function shows some strange behavior with rough meshes, before reproducing 


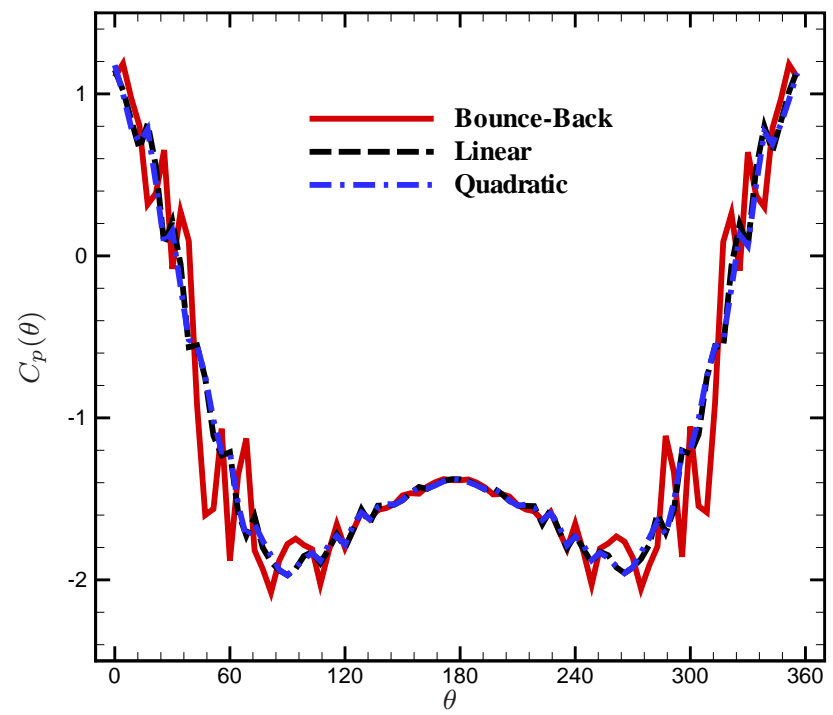

Fig. 7. Flow past a cylinder asymmetrically placed in a channel at $R e=20$. The pressure coefficient $C_{p}(\theta)$ around the cylinder surface is computed with three different boundary conditions. The resolution is $D / \delta_{x}=40 . \theta=0^{\circ}$ is the stagnation point in front of cylinder.

the expected error decay, once the numerical error are small enough to respect the asymptotic analysis.

Table 2

Flow past a cylinder asymmetrically placed in a channel at $\mathrm{Re}=100$. The mesh-size dependence of the Strouhal number St, the maximum drag coefficient $C_{D}^{\max }$ and the maximum lift coefficient $C_{L}^{\max }$. BB, I and II denote the bounce-back, first-order interpolation and second-order interpolation boundary conditions, respectively.

\begin{tabular}{c|c|cccc|c}
\hline \hline & $D / \delta_{x}$ & 10 & 20 & 40 & 80 & NS [32] \\
\hline \multirow{3}{*}{ St } & BB & 0.2778 & 0.2930 & 0.2972 & 0.2979 & \\
& I & 0.2947 & 0.2991 & 0.2993 & 0.2995 & $0.2950-0.3050$ \\
& II & 0.2953 & 0.3000 & 0.2993 & 0.2995 & \\
\hline & BB & 3.951 & 3.329 & 3.241 & 3.266 & \\
$C_{D}^{\max }$ & I & 3.250 & 3.205 & 3.235 & 3.253 & $3.2200-3.2400$ \\
& II & 3.200 & 3.198 & 3.250 & 3.256 & \\
\hline & BB & 0.974 & 0.844 & 0.922 & 1.007 & \\
$C_{L}^{\max }$ & $\mathrm{I}$ & 0.566 & 0.913 & 1.006 & 1.030 & $0.9900-1.0100$ \\
& $\mathrm{II}$ & 0.601 & 0.939 & 1.031 & 1.033 & \\
\hline \hline
\end{tabular}




\subsection{D Flow past a cylinder in an unbounded domain at $R e=100$}

The test case we use to validate the grid stretching method is the $2 \mathrm{D}$ flow past a cylinder in unbounded domain. The flow configuration is illustrated in Fig. 8. We use a computational domain of size $L \times H$. The periodic boundary conditions are applied in the lateral direction $y$. At the inlet, a constant velocity $U$ is imposed. At the outlet, a constant pressure corresponding to $\bar{\rho}=1$ is applied. To minimize effect due to the blockage ratio $D / H$ between the cylinder diameter $D$ and the domain height $H$ on the flow $[52,53]$, we use $D / H=1 / 51$. To minimize the effect from outlet boundary conditions, we use $L=38.5 D$. The cylinder center is located in the middle of the computational domain in $y$-direction, and $13 D$ away from the inlet boundary. With a domain size of $L \times H=38.5 D \times 51 D$, non-uniform mesh would greatly enhance the computational efficiency.

We test the following meshes in our simulations. First, we use a fine mesh with a height of $3 D$ and width of $(4+1+n) D$, of which $4 D$ portion is located upstream to the cylinder front, and $n D$ portion is downstream to the cylinder back, as illustrated by the shaded area around the cylinder in Fig. 8. Outside the fine mesh, grid spacing is stretched exponentially in both directions according to Eqs. (18), with $D$ as the cylinder diameter. We vary the downstream fraction of the fine mesh by varying $n$ to observe the effect due to the fine mesh size in streamwise direction. Similarly, we will also fix the fine mesh length at $(4+1+8) D$, and vary the height of the fine mesh $(2 m+1) D$. All the measurements are made after 100,000 iterations to ensure that the system has reached the periodic state.

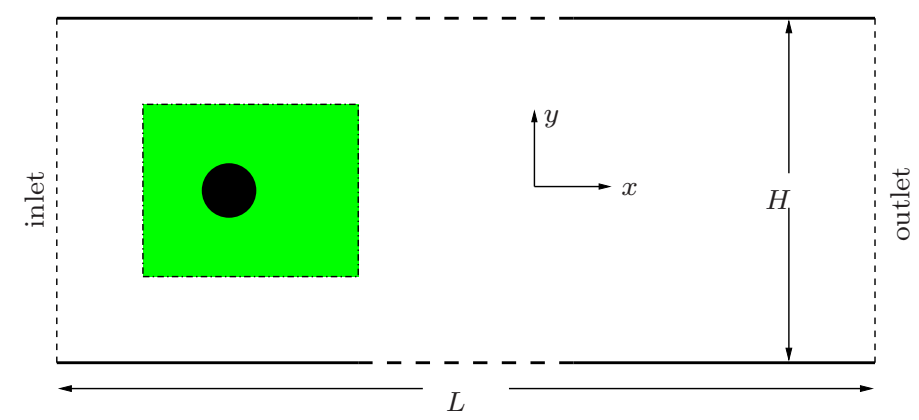

Fig. 8. Computational domain for the flow past a cylinder in unbounded domain. The shaded area around the cylinder is the fine mesh (without grid stretching).

The results of the Strouhal number St, the mean drag coefficient $\bar{C}_{D}$ and the root-mean-square (RMS) lift coefficient $\widetilde{C}_{L}$ are summarized in Table 3, which shows the fine-mesh size dependence of St, $\bar{C}_{D}$ and $\widetilde{C}_{L}$ by varying the fine-mesh length in downstream direction the height in lateral direction. The resolution for the fine mesh is $D / \delta_{x}=40$. Beyond the fine mesh, the grid spacings are stretched according to Eq. (18). We also include the results obtained by the LBE with a uniform fine mesh for the entire domain (LBE) and by a 
Table 3

2D Flow past a cylinder in unbounded domain at $R e=100$. The dependence of the Strouhal number St, the mean drag coefficient $\bar{C}_{D}$ and the RMS lift coefficient $\widetilde{C}_{L}$ on the size of fine mesh in downstream direction $(n D)$ and in the lateral direction $((2 m+1) D)$. Beyond the fine mesh, the grid spacings are stretched according to Eq. (18). The results obtained by the LBE with a uniform fine mesh for the entire domain (LBE) and by a unstructured-grid finite volume Navier-Stokes solver (NS) [38] are also given in the Table.

\begin{tabular}{c|ccccc|cc}
\hline \multirow{2}{*}{$n$} & \multicolumn{5}{|c|}{ The height of fine mesh $=3 D$} & & \\
\cline { 2 - 6 } & 1 & 2 & 4 & 8 & 16 & LBE & NS [38] \\
\hline St & 0.147 & 0.154 & 0.156 & 0.159 & 0.159 & 0.161 & 0.164 \\
$\bar{C}_{D}$ & 1.1713 & 1.3099 & 1.3142 & 1.3241 & 1.3243 & 1.355 & 1.33 \\
$\widetilde{C}_{L}$ & $5.5 \cdot 10^{-5}$ & 0.249 & 0.181 & 0.186 & 0.188 & 0.191 & 0.23 \\
\hline & \multicolumn{1}{|c|}{ The length of fine mesh } & $(4+1+8) D$ & & \\
\cline { 2 - 6 }$m$ & 1 & 2 & 4 & 8 & 16 & & \\
\hline St & 0.1587 & 0.1587 & 0.1590 & 0.1587 & 0.1587 & 0.161 & 0.164 \\
$\bar{C}_{D}$ & 1.3221 & 1.3224 & 1.3223 & 1.3227 & 1.3232 & 1.355 & 1.33 \\
$\widetilde{C}_{L}$ & 0.1841 & 0.1848 & 0.1847 & 0.1853 & 0.1863 & 0.191 & 0.23 \\
\hline
\end{tabular}

unstructured-grid finite volume Navier-Stokes solver (NS) [38] in Table 3. The grid number cover by the uniform fine mesh is $(38.5 \times 40) \times(51 \times 40)=$ $1540 \times 2040 \approx 3.1 \cdot 10^{6}$.

The results in Table 3 clearly show the effect of the size of the fine mesh about the cylinder. Clearly the effect of the fine mesh size diminishes as the fine mesh size enlarges. The results obtained with stretched grids agree well with that obtained with the uniform fine mesh, so long as the size of the fine mesh covering the cylinder is sufficiently large, e.g., $(4+1+8) D \times 5 D=520 \times 200$. However, if the size of the fine mesh is not large enough, e.g., $(4+1+1) D \times$ $5 D=520 \times 200$ for $n=1$, the result of $\widetilde{C}_{L}$ obtained with this mesh is rather inaccurate, as shown in Table 3 . This indicates that one must provide sufficient grid resolution behind the cylinder where vortex shedding takes place. With the fine mesh size fixed at $(4+1+8) D \times 5 D=520 \times 200$ for $n=8$, the grid number of the entire mesh with non-uniformly stretched grids is about $0.5 \times 10^{6}$, which is only about $1 / 16$ of the mesh size of the uniform fine grid. Therefore, the grid stretching method can significantly enhance computational efficiency. Compared to the results obtained by the Navier-Stokes solver [38], the largest difference occurs in the RMS lift coefficient $\widetilde{C}_{L}$, about $17 \%$. Our results also be show that the LBE method is second-order accurate [49]. 


\section{Flows past two tandem cylinders at $\mathbf{R e}=100$}

Our code is written in $\mathrm{C}++$ with a open-source version of the Message Passing Interface library (MPICH 1.3). The numerical simulations presented in this work were carried out on cluster computers available to us at the Department of Computer Science, Old Dominion University (ODU) and Politecnico di Torino.

\subsection{Computational domain, mesh and boundary conditions}

The computational domain for flows past two tandem cylinders of equal diameter $D$ is a rectangle of size $L \times H=(13.5+s+25.5) D \times 47 D$, where $s$ is the dimensionless spacing between two cylinder centers in units of $D$. The distance between the inlet boundary to the first cylinder center is $13.5 \mathrm{D}$, and that between the outlet boundary to the second cylinder center is $25.5 \mathrm{D}$. The cylinders are situated at the centerline of the domain, as illustrated in Fig. 9. A rectangular area of size $(4.5+s+8.5) D \times 5 D$ including both cylinders is covered by a uniform fine mesh, as indicated by the shade rectangle in Fig. 9. The distance between the front boundary of the fine mesh to the first cylinder center is $4.5 \mathrm{D}$, and that between the back boundary of the fine mesh to the second cylinder center is $8.5 \mathrm{D}$. The fine mesh has a height of $5 \mathrm{D}$ and it is placed symmetrically in lateral direction about the domain centerline. The resolution we use is $D / \delta_{x}=40$. A mesh with $s=4$ is depicted in Fig. 10 .

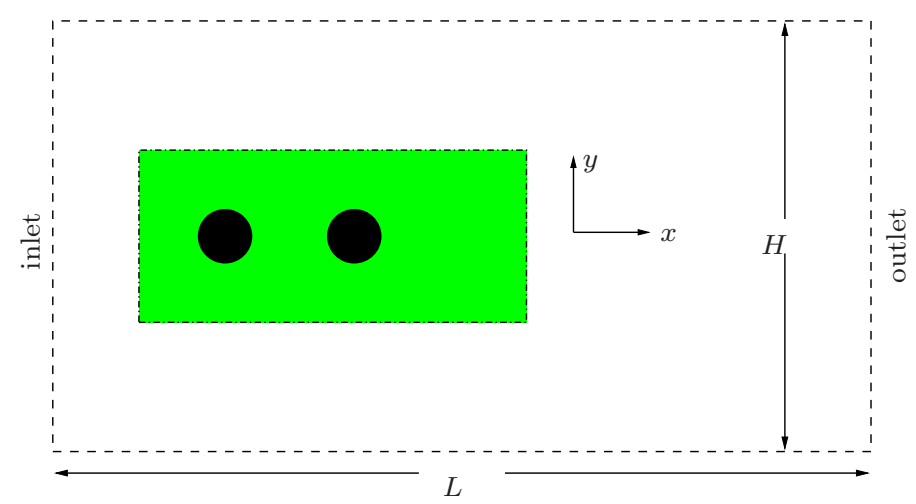

Fig. 9. Schematics of computational domain for the flow past two tandem cylinders. The shaded area of size $(4.5+s+8.5) D \times 5 D$ is covered a uniform fine mesh. The grid stretching is applied to the area outside the fine mesh.

The streamwise direction is along the $x$-axis. The boundary conditions are the same as for the flow past a cylinder in a unbounded domain, i.e., a constant velocity $U$ is enforced at the inlet, a constant pressure condition corresponding to $\rho=1$ is applied at the outlet, and periodic boundary conditions are applied in the boundaries in the $y$ direction. Around the cylinders, second-order interpo- 


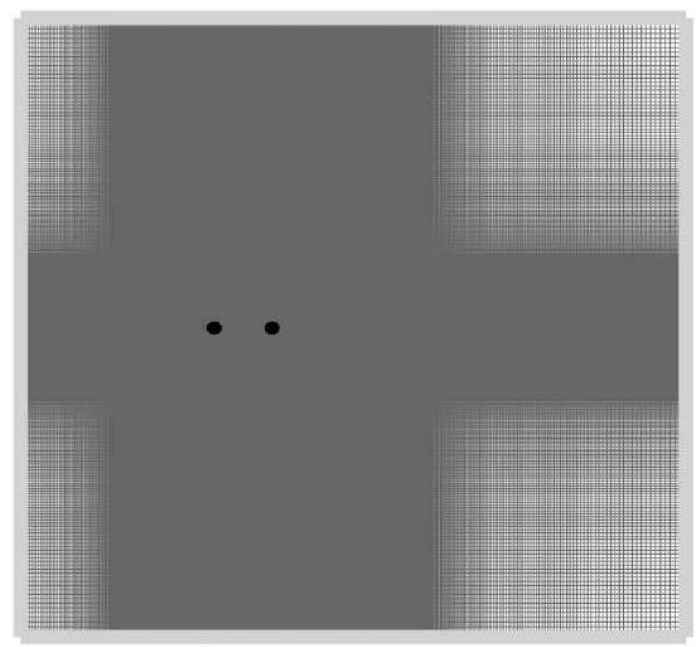

Fig. 10. Non-uniform mesh for the flow past two tandem cylinders with $s=4$. The finest resolution for the mesh covering both cylinders is $D / \delta_{x}=40$.

lated bounce-back boundary conditions are used to achieve no-slip boundary conditions.

The Reynolds number of the flow is based on the inlet velocity $U$ and the cylinder diameter $D$ :

$$
\operatorname{Re}=\frac{U D}{\nu} .
$$

In our simulations, the Reynolds number is fixed at $\mathrm{Re}=100$. We measure the Strouhal number,

$$
\mathrm{St}=\frac{f_{s} D}{U}
$$

where $f_{s}$ is the vortex shedding frequency; and the drag and lift coefficients,

$$
C_{D}=\frac{F_{D}}{\frac{1}{2} \rho U^{2}}, \quad C_{L}=\frac{F_{L}}{\frac{1}{2} \rho U^{2}},
$$

where $F_{D}$ and $F_{L}$ are drag and lift forces, respectively, and $\rho=1$ in our calculations. We study the dependence of St, $C_{D}$ and $C_{L}$ on $s$, the dimensionless spacing between two cylinders.

\subsection{Effect of Mach number}

The LB method is a intrinsically compressible method applied to solve incompressible Navier-Stokes equation, somewhat similar to the method of artificial compressibility [54]. The compressibility, characterized by the Mach number Ma, affects numerical results obtained by using the LBE method, as noted in previous studies [55]. To quantify the effect due to finite Mach number, we conduct a study of Mach number dependence of the drag coefficient $C_{D}$ for the flow past tandem cylinders at $\mathrm{Re}=100$. 
In what follows, we will use the resolution $D / \delta_{x}=40$ and $s=4$, as described in the previous Section. The initial conditions are quiescent velocity field $\boldsymbol{u}_{0}=\mathbf{0}$ and a constant density field $\rho_{0}=1$. The measurements are taken after an initial dimensionless run time $t_{0}^{\prime}=N_{t} U / D=500$, where $N_{t}$ is the number of time steps. We vary the inlet speed $U$ and measure the Mach number dependence of the period $T^{\prime}$, the mean value $\bar{C}_{D}$ and the oscillating amplitude $\Delta C_{D}$ of the drag coefficient for both cylinders. We use $U=0.1 c, 0.05 c$ and $0.025 c$. The results are summarized in Table 4 .

Based on the results of the oscillating period $T^{\prime}$ and the mean drag coefficient $\bar{C}_{D}$ in Table 4 , we can see that the errors in both $T^{\prime}$ and $\bar{C}_{D}$ have approximately a linear dependence on the Mach number Ma. If Richardson extrapolation is applied, we can estimate the asymptotic values of $T^{\prime}$ and $\bar{C}_{D}$ in the limit of $\mathrm{Ma} \rightarrow 0$ to be 3.59000 and 1.20834 , respectively. For $U=0.1 c$, $0.05 c$ and $0.025 c$, the errors of $\bar{C}_{D}$ compared to its estimated asymptotic value 1.20834 are about $7.4 \%, 5.7 \%$, and $3.8 \%$, respectively. That is, the computational time is quadrupled as $U$ is reduced by a factor of four, while the error is only reduced by about half. The results of the oscillating amplitude of $C_{D}$, $\Delta C_{D}$, are much less accurate than $T^{\prime}$ and $\bar{C}_{D}$, because it is a much smaller quantity compared to $\bar{C}_{D}$. Balancing the computational efficiency and accuracy of the results, we will use $U=0.1 c$ for our simulations.

Table 4

The Mach-number dependence of the drag coefficient for the flow past two tandem cylinders at $\operatorname{Re}=100$ and $s=4$. The grid resolution is $D / \delta_{x}=40$ and the mesh is described in Section 4.1. The results are measured after an initial run time $t_{0}^{\prime}:=$ $N_{t} U / D=500$, where $N_{t}$ is the number of time steps. The oscillating period of $C_{D}$, $T^{\prime}$, is given in the unit of $U / D$.

\begin{tabular}{cc|ccc|lll}
\hline \hline$U / c$ & $\mathrm{Ma}$ & $N_{t}$ & $\nu$ & $s_{8}=s_{9}$ & $T^{\prime}$ & $\bar{C}_{D}$ & $\Delta C_{D}$ \\
\hline \hline 0.100 & 0.1732 & 200,000 & 0.04 & 1.61290 & 3.36500 & 1.29770 & 0.03758 \\
0.050 & 0.0866 & 400,000 & 0.02 & 1.78571 & 3.37250 & 1.27718 & 0.02526 \\
0.025 & 0.0433 & 800,000 & 0.01 & 1.88679 & 3.42875 & 1.25484 & 0.02194 \\
\hline \hline
\end{tabular}

\subsection{Results and discussions}

The initial velocity field is zero every where, and the density field is initialized as $\rho=1$ throughout the system. There would be pressure waves generated by initial conditions in the system. In order to avoid the influence of pressure waves caused by unphysical initial conditions, over $2 \cdot 10^{5}$ iterations are carried out before any measurements are taken in our simulations. With $U=0.1 c$ and $D=40 \delta_{x}, N_{t}=200,000$ time steps are equivalent to a dimensionless time $t^{\prime}=N_{t} U / D=500$, which is more than 10 turn-over time corresponding 
to the largest domain with $s=10$. We ensure that the flow field reaches a periodic state before taking measurements [49], and compare our results with the existing ones $[33,37,38]$.

We investigate the dependence of the flow characteristics on the normalized spacing $s$ between two cylinders, for $2 \leq s \leq 10$, at $\operatorname{Re}=100$. With $\operatorname{Re}=100$, the flow is expected to be laminar and periodic [33-38] and two-dimensional [51]. The second cylinder has a synchronization effect on the flow: vortices are shed with the same frequency from both cylinders, the lift forces on both cylinders are in phase, while the drag forces are in anti-phase. As a consequence, the Strouhal number is the same for both cylinders and it characterizes the fluctuation frequency of the flow.

We first show in Fig. 11 the dependence of the Strouhal number St on the dimensionless spacing $s$ between two cylinders. Our LBE results are compared with that of Li et al. [33], Sharman et al. [38] and Mizushima \& Suehiro [37]. Our results are in very good agreement with the existing ones obtained with various Navier-Stokes solvers [33,38,37]. The Strouhal number St in Fig. 11 shows a sharp transition somewhere in $3.0<s<4.0$, which is the most important feature of this flow. The transition of St has been predicted previously [35]. The LBE result indicates that the transition occurs between $3.25<s<3.5$, while the results of Sharman et al. [38] shows the transition happens when $3.75<s<4.0$. This discrepancy is due to the multiplicity of stable solutions in this region, as recently shown by Mizushima and Suehiro [37]. The result of Mizushima and Suehiro [37] shows a hysteresis of $s$-dependence of St, as shown in Fig. 11. As $s$ continues to increase, the value of St increases asymptotically to the value $\mathrm{St} \approx 0.163$ for an isolated cylinder in an unbounded domain.

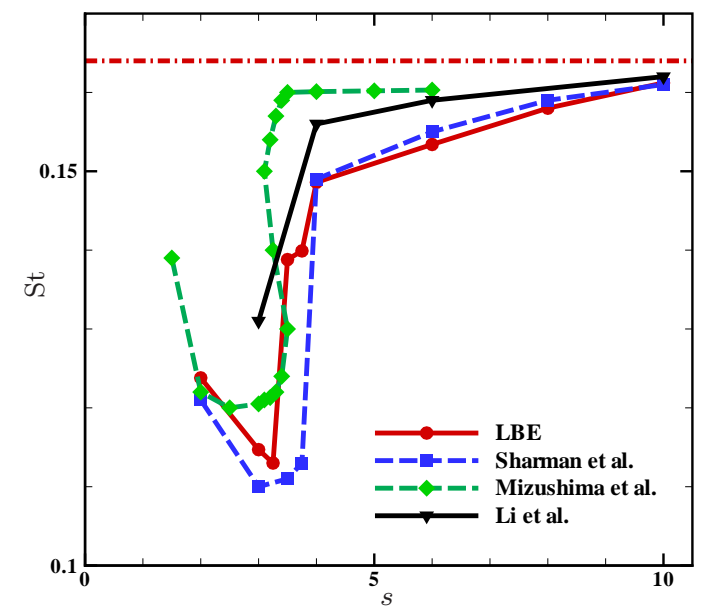

Fig. 11. Flow past two tandem cylinders at $\mathrm{Re}=100$. The dependence of the Strouhal number St on the dimensionless spacing $s$ between two cylinders. The LBE results are compared with that of Li et al. [33], Sharman et al. [38] and Mizushima $\&$ Suehiro [37]. The dash-dot line indicates the value of $\mathrm{St} \approx 0.164$ for a single cylinder in an unbounded domain at $\operatorname{Re}=100$. 
The sharp transition in the Strouhal number St as $s$ varies is due to an instability caused by the flow detached from the first cylinder then interacting with the second one. For small cylinder spacing $s$, the flow separated from the first cylinder re-attaches on the downstream cylinder and vortexes are shed only from the latter. As the spacing $s$ between two cylinders increases beyond certain critical value $s_{c}$, vortexes are shed from both cylinders, thus two perfectly synchronized vortex streets are created in the wake region, as oppose to one vortex street when $s<s_{c}$. In Fig. 12 and we show the streamlines and vorticity field for the flows with $s=2$ and 4. Qualitatively, the two tandem cylinders with $s=2$ behave like a single body, there is only one vortex street generated. When $s=4$, there are clearly two vortex streets generated in the flow.
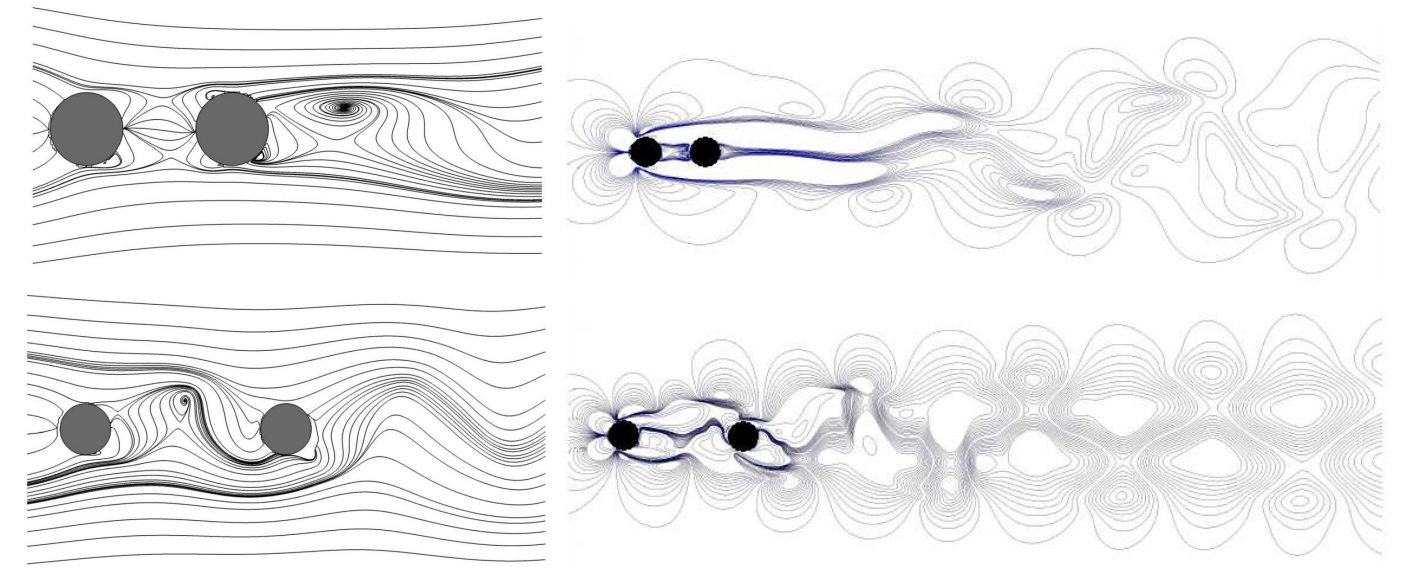

Fig. 12. Flow past two tandem cylinders at $\operatorname{Re}=100$ with $s=2$ (top row) and $s=4$ (bottom row). Instantaneous flow fields: streamlines (left column) and vorticity contours (right column).

Figure 13 shows the mean drag coefficient $\bar{C}_{D}$ for both cylinders as functions of the spacing $s$ between two cylinders. The mean drag coefficient $\bar{C}_{D}$ for the upstream cylinder is larger than that for the downstream one for $2 \leq s \leq 10$. When $s>s_{c}$, the value of $\bar{C}_{D}$ for the first cylinder increases gradually to $\bar{C}_{D} \approx 1.33$ for an isolated cylinder in an unbounded domain; when $s=10$, it is very close to 1.33 . For the second cylinder, the the value of $\bar{C}_{D}$ is much smaller than that of the first, indicating that the suction force due to the first cylinder is rather strong. When $s<s_{c}, \bar{C}_{D}$ for the first cylinder is much smaller than the single-cylinder value $\bar{C}_{D} \approx 1.33$, and $\bar{C}_{D}$ for the second cylinder is even negative, due to strong suction force induced by the first cylinder. Again, a sharp transition in $\bar{C}_{D}$ occurs at $s=s_{c}$.

Figures 14 and 15 show the root-mean-square (RMS) values of the drag and lift coefficients, $\widetilde{C}_{D}$ and $\widetilde{C}_{L}$, respectively, compared with the results of Sharman et al. [38]. The values of $\widetilde{C}_{D}$ and $\widetilde{C}_{L}$ all show a sharp increase at $s=s_{c}$. When $s<s_{c}$, both $\widetilde{C}_{D}$ and $\widetilde{C}_{L}$ for the first cylinder are considerably smaller than their corresponding values for a single cylinder in an unbounded domain. Soon 

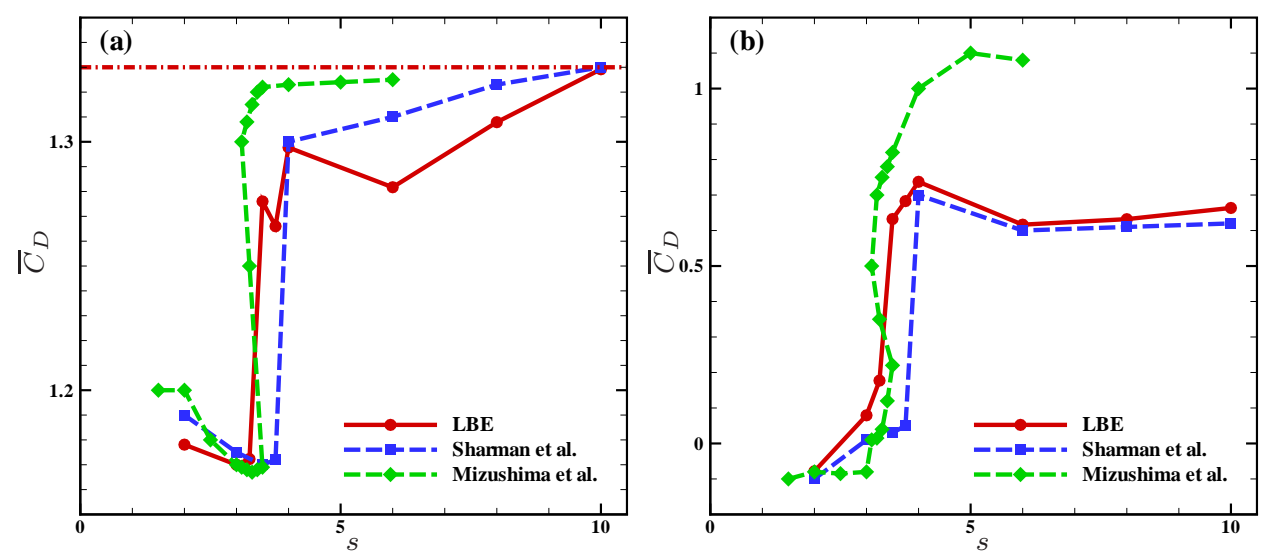

Fig. 13. Flow past two tandem cylinders at $R e=100$. The dependence of the mean drag coefficient $\bar{C}_{D}$ on the dimensionless spacing $s$ between two cylinders. (a) $\bar{C}_{D}$ for the upstream cylinder. The dash-dot line indicates the value of $\bar{C}_{D}=1.33$ for a single cylinder in an unbounded domain at $\operatorname{Re}=100$. (b) $\bar{C}_{D}$ for the down-stream cylinder.

after the transition occurs, both $\widetilde{C}_{D}$ and $\widetilde{C}_{L}$ exceed their corresponding values for a single cylinder and reach their maxima at $s \approx 4$.0, then gradually decrease to their respective single-cylinder values. Both $\widetilde{C}_{D}$ and $\widetilde{C}_{L}$ for the second cylinder behave very similar to that for the first one, i.e., they both encounter a drastic increase at $s=s_{c}$, reach their maxima at $s \approx 4.0$, then gradually decrease to their respective asymptotic values, which should presumably be that for a single cylinder in an unbounded domain as $s \rightarrow \infty$.
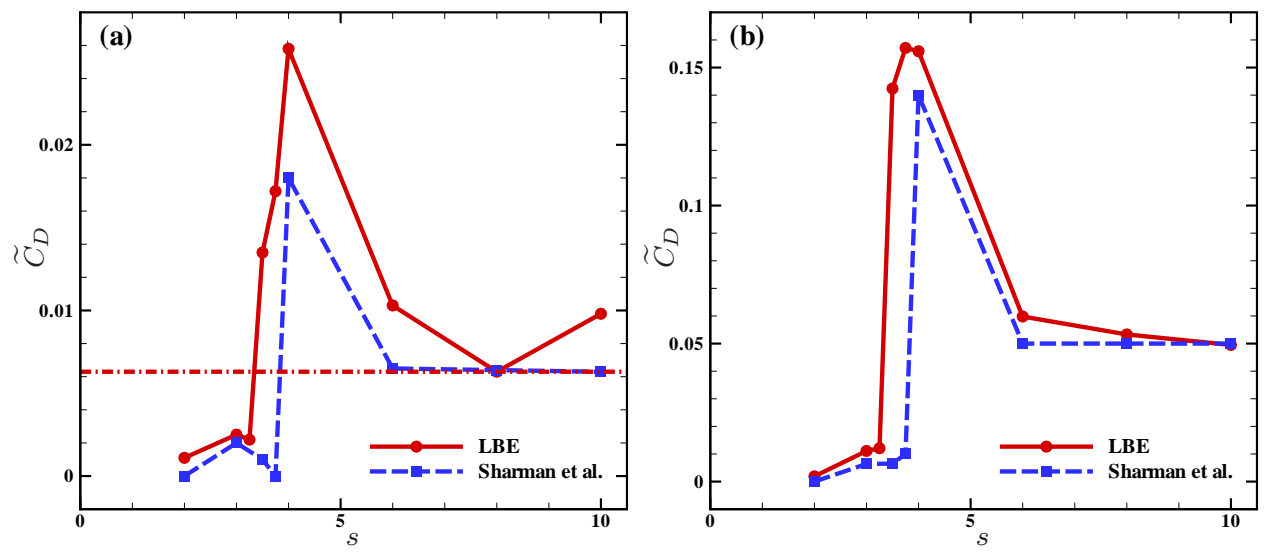

Fig. 14. Flow past two tandem cylinders at $R e=100$. The dependence of the RMS drag coefficient $\widetilde{C}_{D}$ on the dimensionless spacing $s$ between two cylinders. (a) $\widetilde{C}_{D}$ for the upstream cylinder. The dash-dot line indicates the value of $\widetilde{C}_{D} \approx 0.0063$ for a single cylinder in an unbounded domain. (b) $\widetilde{C}_{D}$ for the down-stream cylinder.

Our results shown in Figs. 11, 13-15 quantitatively agree well with the existing results $[33,37,38]$. All the global flow features are quantitatively captured by the LBE simulations. We also notice that there are discrepancies between our results and the ones obtained by various Navier-Stokes solvers $[37,38]$. 

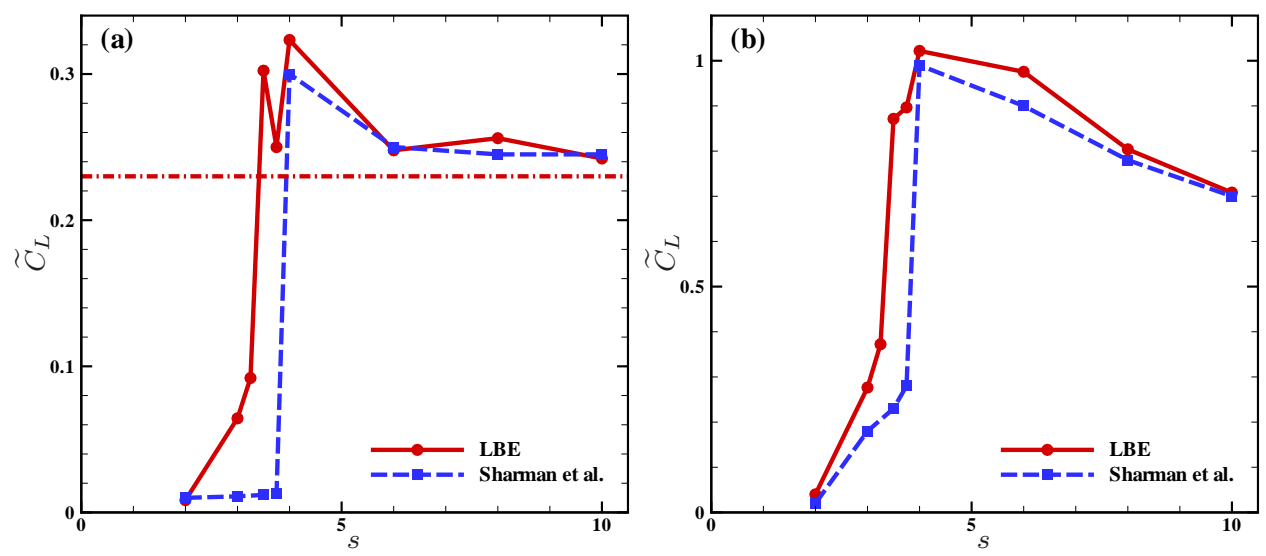

Fig. 15. Flow past two tandem cylinders at $R e=100$. The dependence of the RMS lift coefficient $\widetilde{C}_{L}$ on the dimensionless spacing $s$ between two cylinders. (a) $\widetilde{C}_{L}$ for the upstream cylinder. The dash-dot line indicates the value of $\widetilde{C}_{L} \approx 0.23$ for a single cylinder in an unbounded domain. (b) $\widetilde{C}_{L}$ for the down-stream cylinder.

Compared to the results of Mizushima and Suehiro [37], our results agree better with that of Sharman et al. [38]. Besides the fundamental difference in the solution techniques, we note that body-fitted meshes were used by Sharman et al. [38], and the Cartesian meshes are used in the present work. Also, the discrepancy between our results and that of Sharman et al. [38] may be due to the multiplicity of the solutions near the critical spacing $s_{c}$, as indicated by Figs. 11 and 13.

\section{Conclusions}

In this paper we use the lattice Boltzmann equation with multiple-relaxationtime collision model to simulate laminar flows in two dimensions. For the noslip boundary conditions at flow-solid boundaries, we apply the interpolated bounce-back boundary conditions $[29,8]$. For the inflow and outflow boundary conditions, we use a general bounce-back boundary conditions which can be easily, naturally and consistently realized with the MRT-LBE in particular. To enhance the computational efficiency of the LBE method with uniform meshes, we use the grid stretching method to deal with the non-uniform Cartesian mesh. Even though the techniques we use in the present work are simple and thus easy to implement, the numerical results demonstrate their effectiveness.

The MRT-LBE with non-uniformly stretched Cartesian mesh has been validated by using a number of test cases, including the Poiseuille flow, the flow past a cylinder asymmetrically placed in a channel, and the flow past a cylinder in an unbounded domain. The validated code is used to simulate flows past two tandem cylinders in an unbounded domain at $\mathrm{Re}=100$ with the dimensionless spacing between the cylinders $2 \leq s \leq 10$. Our results agree 
well quantitatively with the existing ones obtained by using the Navier-Stokes solvers.

\section{Acknowledgments}

The authors are grateful to Dr. Meelan M. Choudhari of NASA Langley Research Center for suggesting this problem to them and for his insightful comments of this work, and Prof. Manfred Krafczyk for bringing our attention the effects of the Mach-number on the results obtained by the LBE method. A.M. would like to thank Politecnico di Torino for financial support, and the Department of Mathematics \& Statistics and Center for Computational Sciences at Old Dominion University (ODU) for sponsoring his visit to ODU, between March to July, 2006, during which significant part of this work was accomplished. A.M. would also like to thank Prof. Michele Calì and Prof. Romano Borchiellini of Politecnico di Torino for their support and encouragement of this work. The results reported in this paper are a part of the Master's thesis of A.M. [49]. L.S.L would like to acknowledge the support from the National Science Foundation of the US through the Grant CBET-0500213.

\section{References}

[1] D. Yu, R. Mei, L.-S. Luo, and W. Shyy. Viscous flow computations with the method of lattice Boltzmann equation. Prog. Aerospace Sci., 39:329-367, 2003.

[2] X. He and L.-S. Luo. A priori derivation of the lattice Boltzmann equation. Phys. Rev. E, 55:R6333-R6336, 1997.

[3] X. He and L.-S. Luo. Theory of lattice Boltzmann method: From the Boltzmann equation to the lattice Boltzmann equation. Phys. Rev. E, 56:6811-6817, 1997.

[4] I. Ginzbourg. Boundary Conditions Problems in Lattice Gas Methods for Single and Multiple Phases. PhD thesis, Universite Paris VI, France, 1994.

[5] I. Ginzbourg and P. M. Adler. Boundary flow condition analysis for the threedimensional lattice Boltzmann model. J. Phys. II, 4:191-214, 1994.

[6] I. Ginzburg and D. d'Humières. Local second-order boundary methods for lattice Boltzmann models. J. Stat. Phys., 84:927-971, 1996.

[7] I. Ginzburg and D. d'Humières. Multireflection boundary conditions for lattice Boltzmann models. Phys. Rev. E, 68:066614, 2003.

[8] C. Pan, L.-S. Luo, and C. T. Miller. An evaluation of lattice Boltzmann schemes for porous medium flow simulation. Comput. Fluids, 35(8/9):898-909, 2006. 
[9] D. W. Grunau. Lattice Methods for Modeling of Hydrodynamics. PhD thesis, Colorado State University, Fort Colins, USA, 1993.

[10] P. A. Skordos. Initial and boundary conditions for the lattice Boltzmann method. Phys. Rev. E, 48:4823, 1993.

[11] T. Inamuro, M. Yoshino, and F. Ogino. A non-slip boundary condition for lattice Boltzmann simulations. Phys. Fluids, 7:2928, 1996.

[12] R. S. Maier, R. S. Bernard, and D. W. Grunau. Boundary conditions for the lattice Boltzmann method. Phys. Fluids, 8:1788, 1996.

[13] S. Chen, D. O. Martìnez, and R. Mei. On boundary conditions in lattice Boltzmann methods. Phys. Fluids, 8:2527, 1996.

[14] Q. Zou and X. He. On pressure and velocity boundary conditions for the lattice Boltzmann BGK model. Phys. Fluids, 9(6):1591-1598, 1997.

[15] O. Filippova and D. Hänel. Grid refinement for lattice-BGK models. J. Comput. Phys., 147(1):219-228, 1998.

[16] M. Bouzidi, M. Firdaouss, and P. Lallemand. Momentum transfer of Boltzmann-lattice fluid with boundaries. Phys. Fluids, 13:3452-3459, 2001.

[17] D. d'Humières. Generalized lattice-Boltzmann equations. In B. D. Shizgal and D. P. Weave, editors, Rarefied Gas Dynamics: Theory and Simulations, volume 159 of Prog. Astronaut. Aeronaut., pages 450-458, Washington, D.C., 1992. AIAA.

[18] P. Lallemand and L.-S. Luo. Theory of the lattice Boltzmann method: Dispersion, dissipation, isotropy, Galilean invariance, and stability. Phys. Rev. E, 61:6546-6562, 2000.

[19] D. d'Humières, M. Bouzidi, and P. Lallemand. Thirteen-velocity threedimensional lattice Boltzmann model. Phys. Rev. E, 63:066702, 2001.

[20] D. d'Humières, I. Ginzburg, M. Krafczyk, P. Lallemand, and L.-S. Luo. Multiple-relaxation-time lattice Boltzmann models in three-dimensions. Philos. Trans. R. Soc. Lond. A, 360:437-451, 2002.

[21] M. Junk and W.-A. Yong. Rigorous Navier-Stokes limit of the lattice Boltzmann equation. Asymptotic Anal., 35:165-185, 2003.

[22] M. Junk, A. Klar, and L.-S. Luo. Asymptotic analysis of the lattice Boltzmann equation. J. Comput. Phys., 210(2):676-704, 2005.

[23] M. Junk and Z. Yang. Analysis of the lattice Boltzmann boundary conditions. Proc. Appl. Math. Mech., 3:76-79, 2003.

[24] R. Mei, L.-S. Luo, P. Lallemand, and D. d'Humières. Consistent initial conditions for lattice Boltzmann simulations. Comput. Fluids, 35(8/9):855-862, 2006. 
[25] B. Crouse, E. Rank, M. Krafczyk, and J. Tölke. A LB-based approach for adaptive flow simulations. Int. J. Mod. Phys. B, 17:109-112, 2003.

[26] J. Tölke, S. Freudiger, and M. Krafczyk. An adaptive scheme using hierarchical grids for lattice Boltzmann multi-phase flow simulations. Comput. Fluids, 35(8/9):820-830, 2006.

[27] X. He, L.-S. Luo, and M. Dembo. Some progress in lattice Boltzmann method: Part I. Nonuniform mesh grids. J. Comput. Phys., 129:357-363, 1996.

[28] X. He, L.-S. Luo, and M. Dembo. Some progress in lattice Boltzmann method: Enhancement of Reynolds number in simulations. Physica A, 239:276-285, 1997.

[29] M. Bouzidi, D. d'Humières, P. Lallemand, and L.-S. Luo. Lattice Boltzmann equation on a two-dimensional rectangular grid. J. Comput. Phys., 172:704-717, 2001.

[30] X. He and G. D. Doolen. Lattice Boltzmann method on curvilinear coordinates systems: Flow around a circular cylinder. J. Comput. Phys., 134:306-315, 1997.

[31] X. He and G. D. Doolen. Lattice Boltzmann method on a curvilinear coordinate system: Vortex shedding behind a circular cylinder. Phys. Rev. E, 56(1), 1997.

[32] M. Schäfer and S. Turek. Benchmark computations of laminar flow around a cylinder. In E.H. Hirschel, editor, Flow Simulation with High-Performance Computation II, volume 52 of Notes on Numerical Fluid Mechanics, pages 547$566,1996$.

[33] J. Li, A. Chambarel, M. Donneaud, and R. Martin. Numerical study of laminar flow past one and two circular cylinders. Comput. Fluids, 19(2):155-170, 1991.

[34] M. M. Zdravkovich. Flow Around Circular Cylinders. Volume 2: Applications. Oxford Science Pubblication, Oxford, 2003.

[35] S. Mittal, V. Kumar, and A. Raghuvanshi. Unsteady incompressible flows past two cylinders in tandem and staggered arrangements. Int. J. Numer. Meth. Fluids, 25:1315-1344, 1997.

[36] J. R. Meneghini, F. Saltara, C. L. R. Siqueira, and J. A. Ferrari. Numerical simulation of the flow interference between two circular cylinders in tandem and side by side arrangements. J. Fluid Struct., 15:327-350, 2001.

[37] J. Mizushima and N. Suehiro. Instability and transition of flow past two tandem circular cylinders. Phys. Fluids, 17(10):104107, 2005.

[38] B. Sharman, F. S. Lien, L. Davidson, and C. Norberg. Numerical predictions of low Reynolds number flows over two tandem circular cylinder. Int. J. Numer. Meth. Fluids, 47:423-447, 2005.

[39] L. N. Jenkins, D. H. Neuhart, C. B. McGinley, M. M. Choudhari, and M. R. Khorrami. Measurements of unsteady wake interference between tandem cylinders, 2006. AIAA Paper 2006-3202. 
[40] D. P. Lockard, M. R. Khorrami, M. M. Choudhari, F. V. Hutcheson, and T. F. Brooks. Tandem cylinder noise predictions, 2007. AIAA Paper 2007-3450.

[41] X. He and L.-S. Luo. Lattice Boltzmann model for the incompressible NavierStokes equation. J. Stat. Phys., 88:927-944, 1997.

[42] L.-S. Luo. Comment on "Discrete Boltzmann equation for microfluidics". Phys. Rev. Lett., 92(13):139401, 2004.

[43] R. Mei, D. Yu, W. Shyy, and L.-S. Luo. Force evaluation in the lattice Boltzmann method involving curved geometry. Phys. Rev. E, 65:041203, 2002.

[44] M. Cheng, Q. Yao, and L.-S. Luo. Simulation of flow past a rotating circular cylinder near a plane wall. Int. J. Computat. Fluid Dyn., 20(6):391-400, 2006.

[45] M. Cheng and L.-S. Luo. Characteristics of two-dimensional flow around a rotating circular near a plane wall. Phys. Fluids, 19(6):063601, 2007.

[46] A. J. C. Ladd. Numerical simulations of particulate suspensions via a discretized Boltzmann equation. Part 2. Numerical results. J. Fluid Mech., 271:311-339, 1994.

[47] Y. Peng and L.-S. Luo. A comparative study of immersed-boundary and interpolated bounce-back methods in LBE. Prog. Comput. Fluid Dym., 8(14):156-167, 2008.

[48] H. N. Dixit and V. Babu. Simulation of high Rayleigh number natural convection in a square cavity using the lattice Boltzmann method. Int. J. Heat Mass Trans., 49(3/4):727-739, 2006.

[49] A. Mussa. Numerical simulations of the fluid flow through tandam cylinders by multiple-relaxation-time lattice Boltzmann method. Master's thesis, Politecnico di Torino, Turin, Italy, 2006.

[50] P. Lallemand and L.-S. Luo. Theory of the lattice Boltzmann method: Acoustic and thermal properties in two and three dimensions. Phys. Rev. E, 68:036706, 2003 .

[51] D. J. Tritton. Physical Fluid Dynamics. Oxford Science Publications, Oxford, 1988.

[52] M. Behr, D. Hastreiter, S. Mittal, and T. E. Tezduyar. Incompressible flow past a cyrcular cylinder: Dependence of the computed flow field on the location of the lateral boundaries. Comput. Meth. Appl. Mech. Eng., 123:309-316, 1994.

[53] B. Kumar and S. Mittal. Effect of blockage on critical parameters for flow past a circular cylinder. Int. J. Numer. Meth. Fluids, 50(9):987-1001, 2006.

[54] A. J. Chorin. A numerical method for solving incompressible viscous problems. J. Comput. Phys., 2(1):12-26, 1967.

[55] S. Geller, M. Krafczyk, J. Tölke, S. Turek, and J. Hron. Benchmark computations based on lattice-Boltzmann, finite element and finite volume methods for laminar flows. Comput. Fluids, 35(8/9):888-897, 2006. 